\title{
The Mechanism of Excimer Formation: An Experimental and Theoretical Study on the Pyrene Dimer
}

\author{
Joscha Hoche ${ }^{a}$, Hans-Christian Schmitt ${ }^{a}$, Alexander Humeniuk, Ingo Fischer, ${ }^{*}$ \\ Roland Mitric, and Merle I. S. Röhr* \\ Institut für physikalische und theoretische Chemie, Julius-Maximilians-Universität \\ Würzburg, D-97074, Würzburg, Germany \\ E-mail: ingo.fischer@uni-wuerzburg.de; merle.roehr@uni-wuerzburg.de
}

${ }^{a}$ These authors contributed equally to the work.

\begin{abstract}
The understanding of excimer formation in organic materials is of fundamental importance, since excimers profoundly influence their functional performance in applications such as light-harvesting, photovoltaics or organic electronics. We present a joint experimental and theoretical study of the ultrafast dynamics of excimer formation in the pyrene dimer in a supersonic jet, which is the archetype of an excimer forming system. We perform simulations of the nonadiabatic photodynamics in the frame of TDDFT that reveal two distinct excimer formation pathways in the gas-phase dimer. The first pathway involves local excited state relaxation close to the initial FrankCondon geometry that is characterized by a strong excitation of the stacking coordinate exhibiting damped oscillations with a period of 350 fs that persist for several picoseconds. The second excimer forming pathway involves large amplitude oscillations
\end{abstract}


along the parallel shift coordinate with a period of $\approx 900 \mathrm{fs}$ that after intramolecular vibrational energy redistribution leads to the formation of a perfectly stacked dimer. The electronic relaxation within the excitonic manifold is mediated by the presence of intermolecular conical intersections formed between fully delocalized excitonic states. Such conical intersections may generally arise in stacked $\pi$-conjugated aggregates due to the interplay between the long-range and short-range electronic coupling. The simulations are supported by picosecond photoionization experiments in a supersonic jet that provide a time-constant for the excimer formation of around 6-7 ps, in good agreement with theory. Finally, in order to explore how the crystal environment influences the excimer formation dynamics we perform large scale QM/MM nonadiabatic dynamics simulations on a pyrene crystal in the framework of the long-range corrected tight-binding TDDFT. In contrast to the isolated dimer, the excimer formation in the crystal follows a single reaction pathway in which the initially excited parallel slip motion is strongly damped by the interaction with the surrounding molecules leading to the slow excimer stabilization on a picosecond time scale. 


\section{Introduction}

Excimers, complexes that are only weakly associated in the electronic ground state, but interact strongly when electronically excited, ${ }^{1,2}$ play an important role in the electronic relaxation of molecular aggregates and have also a profound influence on the functional performance of organic devices since they can act as trap sites that limit the exciton diffusion. ${ }^{3-8}$ On the other hand it has been recently found that excimer formation can also have a positive effect on the properties of organic materials, facilitating processes such as singlet fission. ${ }^{9-14}$ For this reason, the understanding of the excimer formation dynamics in molecular aggregates is of fundamental importance for the development of novel materials that can be employed in organic electronics or photovoltaics applications. In addition to their importance for the development of functional materials, excimers can be also employed as probes in biological molecules. ${ }^{15}$ For example, recently long-lived excimers of nanostructures for imaging applications were reported. ${ }^{16}$

While the relation between the structure and spectral properties of multichromophoric assemblies is by now well established, there is a growing experimental evidence that ultrafast nonadiabatic relaxation processes play a fundamental role in determining their functionality. ${ }^{17,18}$ In aggregates such as those formed by self-assembly of $\pi$-conjugated molecular dyes, even a minimal change of the structural parameters can significantly change the efficiency of nonradiative relaxation processes. The coupling between the chromophores in such superstructures can give rise to novel funnels between the delocalized exciton- or charge transfer states that enhance the nonradiative relaxation and limit their functionality, similar to the well established role which conical intersections play in molecular photochemistry. The appearance of such funnels is determined by the interplay between the long-range and shortrange electronic coupling. The latter is critically dependent on the orbital overlap between the building blocks and may lead to ultrafast excimer formation which often competes with the transport and can destroy the function even in seemingly perfect structures. Recently, topologies of molecular aggregates that induce conical intersections on the vanishing points 
of the exciton coupling have been discussed. ${ }^{19}$

In this paper, we undertake a joint theoretical and experimental effort in order to reveal the mechanism of excimer formation in pyrene dimer. This system represents a prototype of an excimer forming aggregate, and it was the first aromatic system for which excimer behavior was observed. ${ }^{20}$ We employ large scale nonadiabatic dynamics simulations in the frame of time-dependent density functional theory (TDDFT) in order to explore the coupled electron-nuclear dynamics that sets in after the electronic excitation and to identify the relaxation channels and the time-scales of relevant processes. In addition to the study of the pyrene dynamics in the gas phase, we also perform simulations on a crystal model which allow us to address the influence of the crystal environment on the excimer formation dynamics. Experimentally, we use picosecond time-resolved photoionization spectroscopy in supersonically cooled jets to study the intrinsic properties of excimers without external perturbations. ${ }^{21}$ Notice that previously the time constants for the excimer formation on the picosecond scale were measured in liquid benzene ${ }^{22}$ but additional phenomena caused by the interaction with the solvent molecules were present that impeded the data interpretation.

From the experimental point of view, excimers are commonly identified by a characteristic red-shifted fluorescence with a lifetime on the order of several ns which has by now been observed for a number of aromatic molecules. For example, in an early gas phase study Saigusa and Lim observed rise times of 36 ps and 46 ps in time-resolved fluorescence studies on fluorene and dibenzofurane clusters and assigned them to excimer formation. ${ }^{23}$ This work demonstrated the power of spectroscopy in free jets for elucidating dynamics of aromatic excimers. However, fluorescence studies lack mass resolution, which is essential in cluster experiments. In this context, the time-resolved photoionization represents a viable alternative. Although it cannot detect the characteristic red-shifted emission, it provides information on cluster size distribution and possibly on cluster fragmentation. It is therefore well suited for real-time studies of intracluster dynamics. ${ }^{24-28}$ Recently the so far most detailed investigation on the excited-state dynamics and excimer formation in molecular dimers 
was reported by Miyazaki and Fujii for the benzene dimer. ${ }^{29}$ These authors extracted time constants of $18 \mathrm{ps}$ and $10 \mathrm{ps}$ for the excimer formation from the $S_{1}$ origin and the $S_{1} 6^{1}$ vibrational level. A relevant factor in experiments on benzene is the fact that the dimer has a T-shaped minimum energy geometry. The excited state dynamics thus corresponds to a rearrangement of a locally T-shaped structure to the parallel sandwich-type structure of the excimer. In contrast to benzene dimer, pyrene dimer exhibits a face-to-face orientation in the electronic ground state that is also characteristic to the arrangement of $\pi$-conjugated molecules in a wide variety of molecular crystals and supramolecular aggregates. Note that the exact mechanism of excimer formation of pyrene is still controversial. While it is generally believed that in solution an excimer is formed in a diffusion-controlled reaction of an excited singlet molecule with a ground-state molecule, ${ }^{30,31}$ the excimer can be also formed by direct excitation of (pre-oriented) ground state dimers. ${ }^{32}$ Recently, in a joint experimental and theoretical study, the formation of excimers in liquid benzene has been assigned to collective excitation of two nearly parallel, thus preoriented monomers. ${ }^{33}$ The aim of our experimental and theoretical study is to provide for the first time the full dynamical picture of the excimer formation mechanism which is initiated by the photoexcitation of an isolated van der Waals dimer containing preoriented pyrene molecules .

\section{Computational methods}

Electronic structure and nonadiabatic dynamics in the gas phase. The electronic structure of the pyrene dimer has been described in the framework of the density functional theory employing the hybrid BH-LYP functional with $50 \%$ Hartree-Fock exchange ${ }^{34-36}$ as implemented in TURBOMOLE $6.3^{37}$ as well as the long-range corrected CAM-B3LYP functional $^{38}$ using Gaussian $09^{39}$ combined with the triple-zeta valence plus polarization (TZVP) basis set ${ }^{40}$ and additionally with the def2-SVP ${ }^{41}$ for a comprehensive comparison. In order to include the dispersion interaction the GD3 correction was used. ${ }^{42}$ Overall, both the BH- 
LYP as well as CAM-B3LYP functional give rise to very similar vertical excitation energies as well as the shape of the potential energy surfaces (see Results). Moreover, in previous studies ${ }^{43}$ it has been shown that the BH-LYP functional reasonably well describes ground and excited state properties of pyrene dimer.

The nonadiabatic dynamics simulations of excimer formation have been performed using our code based on the surface hopping method $^{44}$ in the frame of the linear response time-dependent density functional theory (TDDFT), which has been described in detail in Ref. ${ }^{4-48}$ Here, due to the computational demand, we have employed the efficient BH-LYP functional together with the smaller def2-SVP basis set, which offers a good compromise between accuracy and computational cost. In the following, we provide a brief overview of the the surface hopping dynamics simulations: An ensemble of classical nuclear trajectories is generated in order to simulate the width of the quantum mechanical wavepacket. The initial conditions have been sampled from a 20 ps long ground state MD simulation performed at constant temperature of $50 \mathrm{~K}$ using the Berendsen thermostat. ${ }^{49}$ The temperature of 50 $\mathrm{K}$ has been chosen in order to match the experimental conditions. Each trajectory is then propagated using direct dynamics "on the fly". Along the trajectories the electronic degrees of freedom are propagated by solving the time-dependent Schrödinger equation

$$
i \hbar \dot{c}_{i}(t)=E_{i}(\mathbf{R}(t)) c_{i}(t)-\sum_{j} i \hbar D_{i j}(\mathbf{R}(t)) c_{j}(t),
$$

in the manifold of adiabatic electronic states $E_{i}$ which in the present study contains the ground and the two lowest excited states. Here, $c_{i}(t)$ represents the expansion coefficients of the electronic wavefunction in terms of adiabatic electronic states and $D_{i j}(\mathbf{R}(t))$ represents the nonadiabatic coupling defined by

$$
D_{i j}(\mathbf{R}(t))=\left\langle\Psi_{i}(\mathbf{R}(t)) \mid \frac{d \Psi_{j}(\mathbf{R}(t))}{d t}\right\rangle
$$

The classical Newtonian equations of motion for the nuclear dynamics were solved using the 
velocity Verlet algorithm with a time step of 0.2 fs. In the regions of conical intersections where the character of the electronic wave function changes rapidly due to small nuclear displacements, the nonadiabatic coupling becomes large and might lead to population transfer between the electronic states. In order to simulate the state switching, the hopping probabilities in each nuclear time step are calculated from the change of the electronic state populations $\rho_{i i}=c_{i}^{*} c_{i}$ according to the procedure introduced in Ref. ${ }^{48}$ For this purpose, we decompose the probability for the transition from the state $i$ to the state $j, P_{i \rightarrow j}$ into two independent contributions representing the probability to depopulate the state $i\left(P_{i, \text { depopulation }}\right)$ and the probability to populate the state $j\left(P_{j \text {,population }}\right)$. The probability of depopulation of the state $i$ is given by the rate of change of $\rho_{i i}$

$$
P_{i, \text { depopulation }}=\Theta\left(-\dot{\rho}_{i i}\right) \frac{-\dot{\rho}_{i i}}{\rho_{i i}} \Delta t .
$$

This probability is nonzero only in case that the quantum mechanical population of the $i$ th state is decreasing which is ensured by the Heaviside function $\Theta$ in Eq. (3) defined to be one for positive arguments and zero otherwise. The rate of change $\dot{\rho}_{i i}$ can be obtained directly from the electronic populations in successive nuclear time steps $t$ and $t+\Delta t$ using the finite difference approximation for the derivative. In case of increasing population of some electronic states, the probability to populate the particular state $j$ can be defined as

$$
P_{j, \text { population }}=\frac{\Theta\left(\dot{\rho}_{j j}\right) \dot{\rho}_{j j}}{\sum_{k} \Theta\left(\dot{\rho}_{k k}\right) \dot{\rho}_{k k}} \text {. }
$$

This procedure ensures, that only those states can be populated whose population is growing between two successive nuclear time steps and the probability is defined by the rate of change of the population of the state $j$ normalized to the total rate of change of the populations of all the states whose population is increasing [denominator in Eq. (4)]. Thus, the overall 
probability for hopping from state $i$ to state $j$ can be calculated as

$$
\begin{aligned}
P_{i \rightarrow j} & =P_{i, \text { depopulation }} P_{j, \text { population }} \\
& =\Theta\left(-\dot{\rho}_{i i}\right) \Theta\left(\dot{\rho}_{j j}\right) \frac{-\dot{\rho}_{i i}}{\rho_{i i}} \frac{\dot{\rho}_{j j}}{\sum_{k} \Theta\left(\dot{\rho}_{k k}\right) \dot{\rho}_{k k}} \Delta t .
\end{aligned}
$$

Nuclear velocities were uniformly rescaled in order to conserve the total energy of the systems after a successful state switch. The electronic state population as a function of time is finally obtained by averaging over the ensemble of trajectories.

Nonadiabatic couplings in the framework of TDDFT. The nonadiabatic couplings $D_{i j}$ are obtained from the linear response TDDFT method by using an auxiliary wavefunction for each excited electronic state (for details cf. Ref. ${ }^{46,50}$ ), consisting of all single excitations in the manifold of the occupied and virtual Kohn-Sham (KS) orbitals

$$
\left|\Psi_{i}\right\rangle=\sum_{m}^{v i r t} \sum_{n}^{o c c} c_{m n}^{i}(t) \hat{a}_{m}^{\dagger} \hat{a}_{n}\left|\Phi_{K S}\right\rangle
$$

where the operator $\hat{a}_{m}^{\dagger}$ creates an electron in the virtual orbital $m$ and $\hat{a}_{n}$ annihilates an electron in the occupied orbital $n$. The ket $\left|\Phi_{K S}\right\rangle$ represents the ground state KS determinant, and the coefficients $c_{m n}^{i}$ are determined from the TDDFT eigenvectors. ${ }^{46}$ Employing this wavefunction, the coupling elements are obtained by the finite difference method for the time derivative in the nonadiabatic coupling

$$
\begin{aligned}
D_{i j} & =\left\langle\Psi_{i}(t) \mid \frac{d \Psi_{j}(t)}{d t}\right\rangle \\
& \approx \frac{1}{2 \Delta t}\left(\left\langle\Psi_{i}(t) \mid \Psi_{j}(t+\Delta t)\right\rangle-\left\langle\Psi_{i}(t+\Delta t) \mid \Psi_{j}(t)\right\rangle\right),
\end{aligned}
$$

and can be ultimately reduced to the calculation of atomic overlap integrals as detailed in Ref. ${ }^{45,46,50}$ In order to extend the nonadiabatic simulations to longer time scales which are currently not accessible by the ab initio TDDFT we have also performed simulations in 
the framework of long-range corrected tight-binding TDDFT (lr-TDDFTB) which we have recently developed and implemented ${ }^{51-53}$ together with Grimme's semiempirical dispersion correction. ${ }^{54}$

QM/MM nonadiabatic dynamics in crystal environment. In order to simulate the excited state nonadiabatic dynamics of a pyrene dimer in a crystal a multi-scale approach ${ }^{55}$ with the following setup is used: The pyrene crystal unit cell ${ }^{56}$ is enlarged by duplicating the minimal unit cell along all three axes forming a supercell that contains five pyrene dimers, as presented in Fig. 11.

The total system is partitioned into an inner region containing the central dimer $I$, which is treated quantum mechanically within lr-TDDFTB (QM part) and an outer region $O$, which is described using the DREIDING force field ${ }^{57}$ with periodic boundary conditions (MM part). The total energy is computed using a subtractive scheme:

$$
E=E_{M M}(I+O)+E_{Q M}(I)-E_{M M}(I)
$$

where $E_{M M}$ includes all energy terms of the force field except for the non-bonded electrostatic term. Since hydrogen and carbon have similar electronegativities the partial charges in pyrene are small, so that the electrostatic interactions in the MM calculations can be neglected. The dominant effects of putting the pyrene dimer into a crystal environment are steric constraints on the parallel shifting motion of the central dimer. While electrostatic embedding is important for describing the interaction with polar solvents such as water, mechanical embedding should be sufficient for the rigid, non-polar pyrene molecules. The crystal is held together by pi-stacking and not ionic forces. The small polarization of the QM part by the partial charges in the MM part is also ignored. In the MM calculation periodic boundary conditions are applied so that atoms on one end of the supercell are in contact with atoms on the opposite side through bonding or non-bonding interactions. This ensures that the shape of the periodic supercell is not distorted during the dynamics. The electronic 
structure of the inner region is described using non-periodic lr-TDDFTB, so discrete electronic energy levels result rather than continuous bands, which are present in a crystal. The initial conditions for 50 trajectories were generated by assigning random velocities to the nuclei. The resulting trajectories were propagated for 1 ps on the ground state at a constant temperature of $50 \mathrm{~K}$ using a Berendsen thermostat to obtain an equilibrated ensemble. Then all trajectories were lifted adiabatically to the $S_{1}$ state and allowed to evolve for another 5 ps, but now at constant energy. This simulates the situation where the central pyrene dimer is excited and all other dimers are in the ground state. No electronic coupling exists between the different dimers but vibrational energy can flow from the central dimer to the crystal.

\section{Experimental methods}

Pyrene was obtained commercially from Sigma-Aldrich and used without further purification. The samples were placed in an oven attached to a pulsed solenoid valve and heated to around $160{ }^{\circ} \mathrm{C}$. The evaporated pyrene molecules were seeded in Argon and expanded into a differentially pumped vacuum chamber through a $1.0 \mathrm{~mm}$ diameter nozzle to obtain a cooled molecular beam (temperature around $50 \mathrm{~K}$ ). The skimmed jet entered the experimental chamber with a pressure of $5.0-8.5 \times 10^{-7}$ mbar. The detailed setup of the apparatus has

been described in a previous publication. ${ }^{58}$ In the experimental chamber the molecules were ionized by a picosecond laser with a repetition rate of $10 \mathrm{~Hz}$. The details of the laser system, consisting of a solid state Nd:YLF (neodymium-doped yttrium lithium fluoride) laser and an optical parametric generator (OPG), are also given elsewhere. ${ }^{59}$ In most experiments described below the tunable OPG output was used as the pump beam, while a small part of either the $2^{\text {nd }}\left(18975 \mathrm{~cm}^{-1}, 2.353 \mathrm{eV}, 527 \mathrm{~nm}\right)$ or $3^{\text {rd }}\left(28490 \mathrm{~cm}^{-1}, 3.532 \mathrm{eV}, 351 \mathrm{~nm}\right)$ harmonic of the Nd:YLF laser $(200-300 \mu \mathrm{J})$ was sent over a motorized linear translation stage and used as the probe pulse. The OPG output between 27000 and $33000 \mathrm{~cm}^{-1}$ yielded pulses with a bandwidth of approx. $20-25 \mathrm{~cm}^{-1}$ and a pulse energy of 30 to $150 \mu \mathrm{J}$. The 
instrument response function (IRF) was determined to be around 4 ps. Measurements at an excitation wavelengths of $351 \mathrm{~nm}$, which is the point of degeneracy of the OPG, were conducted using the Nd:YLF laser $3^{\text {rd }}$ harmonic as the pump and the $2^{\text {nd }}$ harmonic as the probe. Pump and probe pulses were overlapped in front of the experimental chamber using a dichroic mirror. Unfocused pulses yielded the best signal-to-noise ratio (SNR). Ions were accelerated in a TOF mass spectrometer onto a multichannel plate (MCP) detector. The data were averaged for at least 50 shots per point for every scan, 5 to 15 scans were averaged to receive a good signal-to-noise ratio.

\section{Results and discussion}

REMPI-spectra. Fluorescence excitation spectra of pyrene and its dimer have been recorded before in free jets. ${ }^{60,61}$ The origin of the $S_{1}\left({ }^{1} B_{2 u}\right) \leftarrow S_{0}\left({ }^{1} A_{1 g}\right)$ transition of the monomer was observed at $27220 \mathrm{~cm}^{-1}$ (corresponding to $3.375 \mathrm{eV}$ ), ${ }^{60,62}$ while the origin of the strongly vibronically coupled $S_{2}$ state, is observed at $31100 \mathrm{~cm}^{-1}(3.856) .{ }^{60}$ The dimer $(\mathrm{Py})_{2}$ exhibits a broad and unstructured electronic spectrum that sets in around $27030 \mathrm{~cm}^{-1} \cdot{ }^{61}$ These earlier frequency-domain studies guided the present time-domain experiments.

Resonance-enhanced multiphoton ionization spectra (REMPI) of pyrene monomer were recorded by tuning the picosecond OPG. The adiabatic ionization energy (IE) of neutral pyrene was determined to be $59888 \mathrm{~cm}^{-1}(7.425 \mathrm{eV}),{ }^{63}$ so the molecule can be ionized in a $[1+1]$ process. Two selected spectra, corresponding to the origin regions of the $S_{1}\left({ }^{1} B_{2 u}\right) \leftarrow$ $S_{0}\left({ }^{1} A_{1 g}\right)$ and the $S_{2} \leftarrow S_{0}$ transition are given as Fig. S1 and S2 in the electronic supplementary information (ESI). As can be seen, individual transitions into low-lying vibrational bands of the $S_{1}$ state can be resolved. They have been assigned before to $a_{g}$ and vibronically induced $b_{3 g}$ vibrations. ${ }^{60}$ The close-lying bands at the $S_{2}$ origin around $31055-31250 \mathrm{~cm}^{-1}$, however cannot be separated due to the $20-25 \mathrm{~cm}^{-1}$ bandwidth of our ps-laser system. In the dimer mass channel only a broad band, starting around $27030 \mathrm{~cm}^{-1}$ was observed (see 
Fig. 1), again in agreement with earlier work. In Fig. 1 we also compare the experimental spectrum with theoretical simulations for a thermal ensemble that has been used to run nonadiabatic dynamics simulations (see below). As can be seen, the onset of the absorption as well as the shape of the first peak are almost perfectly reproduced by theoretical simulations at the TDDFT/BH-LYP level of theory. At higher wavenumbers above $31000 \mathrm{~cm}^{-1}$ $(3.844 \mathrm{eV})$ the experimental spectrum shows transitions into higher-lying electronic states that are not included in the calculations.

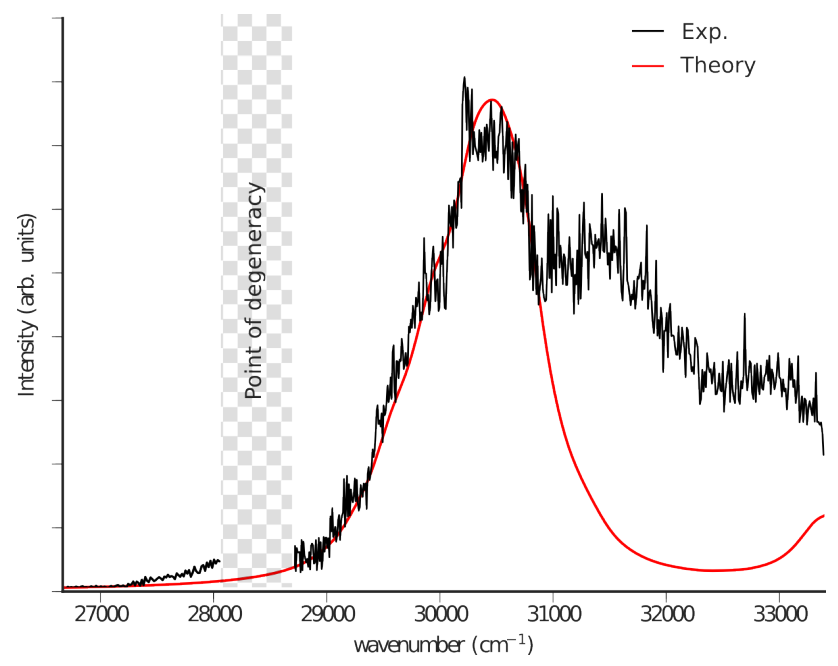

Figure 1: $[1+2]-R E M P I$ of the pyrene dimer and simulated absorption spectra (red), shifted by $1290 \mathrm{~cm}^{-1}(0.16 \mathrm{eV})$ to lower energies to match the experimental maximum absorption peak. A broad band starting around $27000 \mathrm{~cm}^{-1}$ is visible. Note that the point of degeneracy of the ps laser-system used in this study is located at $28490 \mathrm{~cm}^{-1}$. Due to the low laser power no signals could be recorded around this point.

Mass spectra. A challenge in all cluster experiments is the finite size distribution one generally has to deal with. When the electronic spectrum is sufficiently resolved, one can often ionize a particular cluster size selectively. For clusters with broad and unstructured bands this is not possible. As fragmentation of larger clusters can obscure signals in the lower mass channels, it is necessary to select a narrow size distribution by choosing suitable expansion conditions, i.e. by varying the sample temperature and the seed gas pressure. 


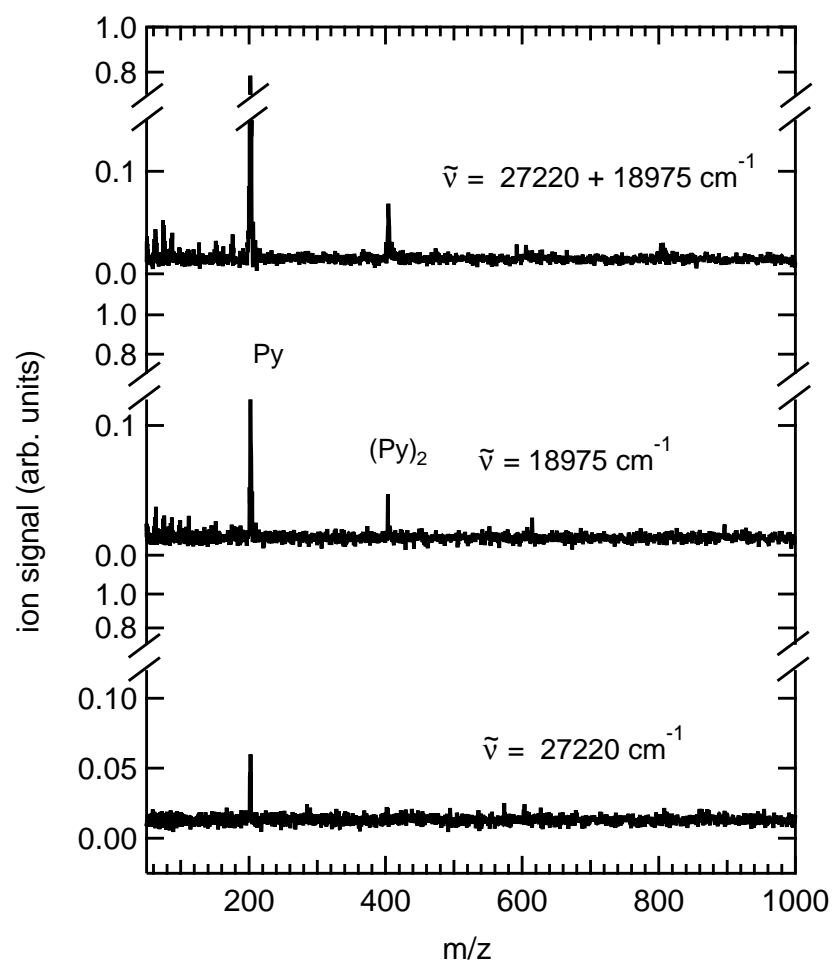

Figure 2: Mass Spectra of pyrene, recorded at zero time delay between pump and probe laser (top trace). All spectra are dominated by the monomer. At $18975 \mathrm{~cm}^{-1}$ (probe only) there is a one-color background signal in the dimer mass channel (center trace). At 27220 $\mathrm{cm}^{-1}$ (pump only) the cluster background signal is negligible (bottom trace).

Fig. 2 shows mass spectra that were recorded at $27220 \mathrm{~cm}^{-1}$, close to the $S_{1}$ origin of the pyrene monomer. The second harmonic of the Nd:YLF laser at $18975 \mathrm{~cm}^{-1}$ was chosen as the ionization pulse. As can be seen, the monomer signal dominates the mass spectrum under all conditions. A dimer signal is clearly visible, while higher clusters appear with negligible intensity. The probe only $\left(18975 \mathrm{~cm}^{-1}\right)$ spectrum yields a background signal due to multiphoton ionization. Small signals at low masses indicate that some dissociative photoionization is present. The pump only $\left(27220 \mathrm{~cm}^{-1}\right)$ spectrum on the other hand shows no background from clusters. Mass spectra recorded at the absorption maximum of the dimer at $29850 \mathrm{~cm}^{-1}$ excitation are given in the ESI (Figure S9). They show in addition to the monomer and the dimer a substantial trimer signal.

Experiments at the $S_{2}$ Origin of the Pyrene Monomer. The spectrum around the 
$S_{2}$ origin (see Fig. S2 for a wavelengths scan) is considered to be an example for intermediate level structure, i.e. an individual bright zero order state of $S_{2}$ (for example the $S_{2}$ origin band) is coupled to a finite number of vibrational levels in $S_{1}$. An extensive simulation of the fluorescence excitation spectrum, taking vibronic coupling into account has already been presented. ${ }^{62}$ Due to the bandwidth of our ps-laser several eigenstates (probably five to ten) are excited around $31190 \mathrm{~cm}^{-1}$. The pump-probe signal (see Fig. S3) decays rapidly to a constant level. A fit to the data yields $3.1 \mathrm{ps}$. Given the 4 ps IRF of our setup, this is the ultimate limit for a time constant that can be extracted from our experiments. The signal decay is due to the redistribution of vibrational energy (IVR) from the $S_{2}$ zero order state to the first tier of $S_{1}$ levels as has been described in. ${ }^{60}$

Time-Delay Scans of the Pyrene Dimer. After characterizing the spectrum by MPI, we recorded time-delay scans at various wavelengths to gain insight into the dynamics of the pyrene dimer, $(P y)_{2}$. As the clusters show broad and unstructured absorption bands, selective excitation of one mass is not possible. Under these circumstances dissociative photoionization is a major obstacle in cluster experiments, because dynamics in a larger cluster can erroneously appear in a lower mass channel as discussed previously. ${ }^{27}$ It is therefore necessary to discuss the relevant ionization and fragmentation thresholds, which are summarized in Figure S10. In a recent synchrotron radiation photoionization experiment ${ }^{64}$ IEs of $6.95 \mathrm{eV}$ and $6.76 \mathrm{eV}$ were determined for the pyrene dimer and trimer. For the appearance energies of the clusters ${ }^{65}$ values of $\mathrm{AE}\left(P y_{2}, P y^{+}\right)=8.7 \mathrm{eV}$ and $\mathrm{AE}\left(P y_{3}, P y_{2}^{+}\right)=7.9 \mathrm{eV}$ were found. We therefore started our investigation with a pump wavenumber of $27220 \mathrm{~cm}^{-1}$ (corresponding to $3.375 \mathrm{eV}$ or $367.4 \mathrm{~nm})$ and a probe wavenumber of $18975 \mathrm{~cm}^{-1}(2.353 \mathrm{eV}$ or $527 \mathrm{~nm})$. Assuming a $\left[1+2^{\prime}\right]$ MPI scheme the total energy amounts to $8.08 \mathrm{eV}$ and lies between $\operatorname{IE}\left(P y_{2}\right)$ and $\operatorname{AE}\left(P y_{2}, P y^{+}\right)$, thus the dimer is ionized, but dissociative ionization leading to a charged monomer is suppressed. Note that the pump energy is very close to the band onset and the absorption cross section is small. 


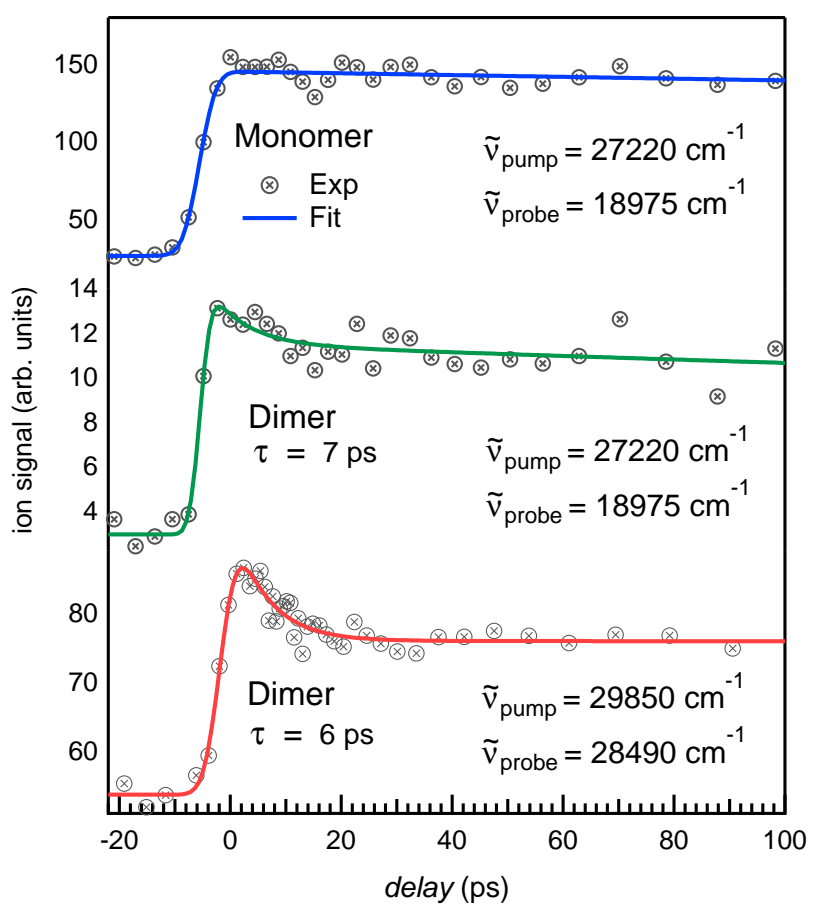

Figure 3: Selected time delay scan. Close to the $S_{1}$ band origin, the pyrene monomer shows a long lifetime (blue line), while the dimer (green line) exhibits a picosecond transient. This transient, which decays to a constant offset, becomes more pronounced at $29850 \mathrm{~cm}^{-1}$ excitation (red line), close to the absorption maximum of the dimer.

Fig. 3 shows selected time delay scans. The monomer signal (top trace, blue line) shows a step function, in agreement with the nanosecond lifetime reported previously, thus fragmentation of the dimer is successfully suppressed. The dimer signal in the center trace (green line) exhibits an initial decay with a time constant $\approx 7$ ps to a relatively large offset. When the probe beam was slightly focused, signal levels increased significantly, but considerable fragmentation became visible. Note that the total energy in a $\left[1+2^{\prime}\right]$ scheme is slightly above $\mathrm{AE}\left(P y_{3}, P y_{2}^{+}\right)=7.9 \mathrm{eV}$, so dissociative ionization of the trimer is in principle possible. It would however lead to a signal increase in the dimer channel with time. Nevertheless it is therefore important to reduce the concentration of larger clusters in the beam as much as possible. As visible in Fig. 2, the trimer signal is small under our experimental conditions. At lower pump energies $\left(\tilde{\nu} ; 27220 \mathrm{~cm}^{-1}\right)$ signal levels were too low to record pump-probe spec- 
tra with sufficient signal to noise $(\mathrm{S} / \mathrm{N})$ ratio. The maximum of the dimer absorption band is measured around $29850 \mathrm{~cm}^{-1}(3.701 \mathrm{eV})$. We therefore conducted further time-resolved experiments at this pump energy, using a $28490 \mathrm{~cm}^{-1}$ probe $(3.532 \mathrm{eV})$. As the dimer itself absorbs substantially at $28490 \mathrm{~cm}^{-1}$, the probe beam had to be attenuated until the background signal was negligible. This $\left[1+1^{\prime}\right]$ process deposits a total energy of $7.23 \mathrm{eV}$ in the cluster, so a dissociative ionization of the trimer is not possible, in agreement with the energetics displayed in Figure S10. Therefore the $P y_{3}$ signal visible in the mass spectrum (see ESI) is not expected to perturb the resulting time-delay scan, depicted in the bottom trace of Fig. 3. The initial transient is more pronounced than at $27220 \mathrm{~cm}^{-1}$, but the time constant of the fit (red line) is only slightly shorter, around 6 ps. Again the signal decays to a large offset level.

We will in the following discuss the possible reasons for this transient in the dimer channel. As reasoned above, dissociative photoionization of the dimer or trimer can be neglected as the source of the transient, because the total energy of the $\left[1+1^{\prime}\right]$ process lies below $\mathrm{AE}\left(P y_{3}, P y_{2}^{+}\right)$and $\mathrm{AE}\left(P y_{2}, P y^{+}\right)$, see Figure S10. A second source could be molecular rotation. Therefore further experiments were carried out at $28490 \mathrm{~cm}^{-1}$ pump, employing probe energies of $25000 \mathrm{~cm}^{-1}$ and $18975 \mathrm{~cm}^{-1}$. The two spectra recorded with perpendicular and parallel polarization of pump- and probe-laser are given in Fig. S4 and show very similar lifetimes of 6-7 ps, which indicates that the observed transient is not related to molecular rotation. Furthermore, the large signal offset at long delay times rules out ionization from the electronic ground state, which would be associated with a small ionization probability, because Franck-Condon factors for ionization from highly excited $S_{0}$ vibrational states are negligibly small at the ionization threshold. Therefore internal conversion to the ground state can also be disregarded as a deactivation pathway. The observed picosecond dynamics has thus to be linked with dynamics within the electronically excited states that leads to a small change in the absorption probability. Intersystem crossing (ISC) would be a possibility, but the 6-7 ps time constant is unusually fast for ISC in a pure hydrocarbon. For example, 
for the pentacene dimer hosted in a p-terphenyl crystal the rate constant for ISC was found to be $8.8 \times 10^{-7} \mathrm{~s}^{-1} \cdot{ }^{66}$ Formation of an excimer, connected with a reorientation of the two pyrene units is a more likely alternative. As the excimer is a lower energy isomer, there is excess energy available which is redistributed as vibrational energy in the cluster. At the low probe energies chosen to suppress dissociative photoionization, the ionization probability of vibrationally excited dimers is reduced, leading to a decreasing dimer ion signal. However, ionization from the excimer is still possible, resulting in a rather large offset at long delay times. As visible in Fig. 3 the transient is more pronounced at $29850 \mathrm{~cm}^{-1}$ excitation and the offset level is lower as compared to $27220 \mathrm{~cm}^{-1}$, because the available excess energy in the probe step is smaller and the ionization probability of the excimer is reduced compared to the $\left[1+2^{\prime}\right]$ experiments at $27220 \mathrm{~cm}^{-1}$.

Interestingly similar time constants as in the present work were observed for emission changes from zeolite-incorporated pyrene (7-13 ps), ${ }^{67}$ and in pyrene-doped polymers (4-10 ps). ${ }^{68}$ In both cases these contributions were assigned to excimer formation in the respective systems. The present work on the isolated pyrene dimer seems to confirm this interpretation. We therefore carried out theoretical work to investigate the intrinsic dynamics of the pyrene dimer in more detail and validate the qualitative interpretation given above.

Structural properties and excited states in pyrene-dimer. The lowest energy isomer of the pyrene dimer in the ground electronic state $\left(S_{0}\right)$ exhibits a stacked structure with the distance between the molecular planes of $3.38 \AA$ (cf. Fig. ??a) calculated at the BHLYP/def2-SVP+GD3 level of theory. In the lowest energy structure the monomers are displaced along the long molecular axis leading to the structure with $C_{2 h}$ symmetry shown in Fig. ??a. In the following, this structure will be referred to as the parallel-displaced (PD) structure. In the Fig. ??b we also provide the lowest energy structure in the first excited state that corresponds to the excimer with the interplanar distance of 3.27 Åexhibiting $D_{2 h}$ symmetry. Upon stacking, the electronic interaction between the individual monomers leads to the formation of molecular orbitals that are delocalized over both subunits. The 
frontier molecular orbitals for both geometries can be derived from the frontier orbitals of the monomers and are shown in Fig. ??a and b together with their decomposition in terms of the monomer orbitals. Since the distance between the monomers is relatively small, the interaction between the monomer MOs leads to the relatively large energy splitting of the dimer orbitals. Overall, from the HOMO and LUMO orbitals of both monomers four frontier orbitals arise. The HOMO-1 and HOMO orbitals are represented by a negative and positive linear combination of the HOMO orbitals of both monomers. Notice that the HOMO-1 is destabilized with respect to the monomer due to the largely antibonding interaction in the PD structure while the HOMO orbital is stabilized. This situation reverses in the stacked structure, where the dimer HOMO is strongly destabilized and the HOMO-1 is stabilized. As can be seen, the same trend applies also to the unoccupied molecular orbitals. Thus, the parallel shift along the long molecular axis from the PD structure to the fully stacked structure leads to the overall lowering of the HOMO-LUMO gap. The excitation energies presented in Table S1 show good accordance between the results obtained using the BHLYP and CAM-B3LYP functionals and are in satisfactory agreement with the experimentally observed ones and the theoretical values from Ref. ${ }^{69}$ In order to explore the change in the energies and intensities of the lowest excited states we have calculated sections from the potential energy surfaces along the interplanar distance coordinate $\left(R_{z}\right)$, shown in Fig. S11 and along the parallel shift coordinate $\left(R_{x}\right)$ which are presented in Fig. 5. The same sections from the potential energy surfaces in the framework of CAM-B3LYP/def2-SVP are given in Fig. S8 and are in good agreement with the results obtained at the BH-LYP level. As indicated by the natural transition orbitals (NTOs) given in Fig. S6 and S7 for the Franck-Condon geometry, the two lowest excited states $\left(S_{1}\right.$ and $\left.S_{2}\right)$ are almost degenerate and exhibit dominantly Frenkel-exciton character, i.e. they can be understood as linear combination of the local excitations of the individual monomers. The corresponding NTOs are delocalized over the whole dimer. (denoted B in Fig. 5).

The character of the two lowest excited states can also be seen in the $S_{0} \rightarrow S_{1}$ transition 


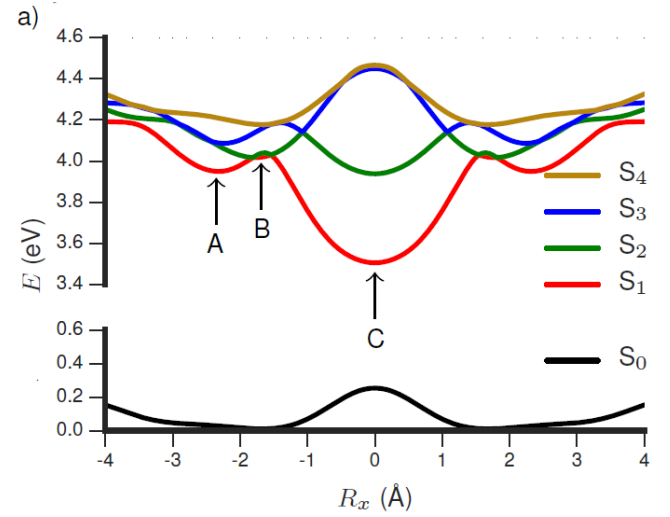

b)

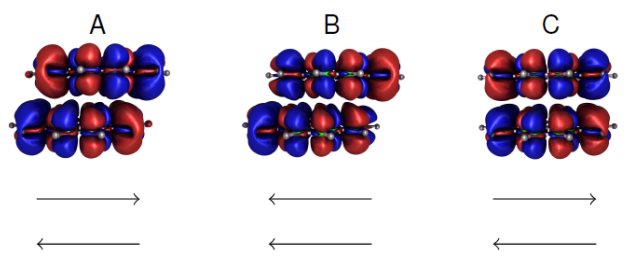

c)
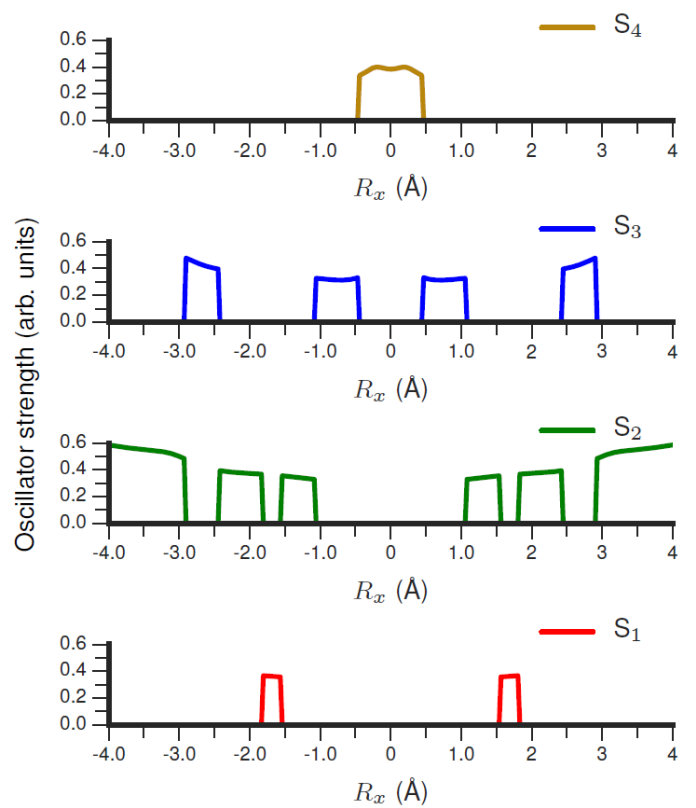

Figure 5: (a) Energies of the ground $\left(S_{0}\right)$ and the four lowest $\left(S_{1}-S_{4}\right)$ excited states of the pyrene dimer along the parallel shift coordinate $\left(R_{x}\right)$ at an interplanar distance $\left(R_{z}\right)$ of $3.3 \AA$. The coordinate system is shown in the inset.(b) The transition densities between the ground state and the first excited states for three selected geometries denoted with A, B, C are shown in the lower part of the figure illustrating the change between the parallel and antiparallel arrangement of the monomer transition dipoles along the $R_{x}$ coordinate. (c) Oscillator strength for the $S_{0} \rightarrow S_{i} i=1-4$ transition along the $R_{x}$ coordinate illustrating the change of the diabatic character of the excited states. 
densities calculated for three characteristic geometries along the parallel shift coordinate $\left(R_{x}\right)$ denoted $\mathrm{A}, \mathrm{B}$ and $\mathrm{C}$, in Fig. 5b. The local transition dipole moments of the individual monomers are parallel at the FC geometry (point B) corresponding to a bright $S_{1}$ state. As the two monomers slide along the parallel direction the diabatic character of the $S_{1}$ and $S_{2}$ states interchange at two geometries close to point $\mathrm{B}$ which indicates the presence of a conical intersection. The lowest excited state changes from the parallel to the anti-parallel orientation of the transition dipole moments as can be seen from the transition densities at points $\mathrm{A}$ and $\mathrm{C}$. At the perfectly stacked geometry (point $\mathrm{C}$ ) the splitting of the excitonic state manifold is much larger than at the FC geometry and has a value of almost $0.5 \mathrm{eV}$. In order to illustrate the change of the electronic character of the lowest states the oscillator strengths of the four lowest excited states as a function of the parallel displacement are shown in the right hand side of Fig. 5. While either the $S_{1}$ and $S_{2}$ states are intense close to the FC geometry, the parallel displacement towards the stacked structure leads to the exchange of the intensities such that close to the perfectly stacked structures the higher lying states $S_{3}$ and $S_{4}$ become bright. In fact, the perfectly stacked structure is an example of the H-aggregate in which the highest lying exciton state has the whole intensity while the initial PD structure is an intermediate case between the J- and $\mathrm{H}$-aggregate. The crossings between the electronic states that arise along the parallel shift coordinate $\left(R_{x}\right)$ may act as funnels that mediate the electronic relaxation since strong nonadiabatic couplings will occur close to the crossing geometries mediating efficient nonradiative transitions. In the following, we will address the question how the conical intersections between excitonic states may arise as a combining effect of the long-range and short-range electronic couplings in the diabatic picture.

\section{Conical intersections within the exciton model for the excited states of pyrene}

dimer. Although all calculations and dynamics simulations have been performed using the adiabatic states of the dimer, in this section we rationalize the excited states of the pyrene dimer in terms of an exciton model. The shape of the potential energy surfaces shown in Fig. 
5, the energetic splitting and the electronic character of the excited states can be understood in terms of a minimal model in which the excited states of the dimer are formed by coupling between the local (diabatic) excited states of the monomers and the intermolecular charge transfer states. Since the two monomers are very close together, the overlap between the electronic densities cannot be neglected so that in addition to the long-range Förster type of electronic coupling also the short-range electronic coupling, which is strongly dependent on the orbital overlap, needs to be taken into account. It has been previously shown that the short-range couplings are particularly important in flat molecules such as polycyclic aromatic hydrocarbons ${ }^{70,71}$ that can be tightly packed.

The short-range electronic coupling contains the contribution of the Dexter-type exchange interaction as well as the coupling to the intermolecular charge transfer states. A detailed analysis of the electronic couplings in stacked $\pi$-systems has been provided already long time ago. ${ }^{72}$ The coupling can thus be decomposed in a short- and long-range contribution according to,

$$
V=V_{L R}+V_{S R}
$$


a)

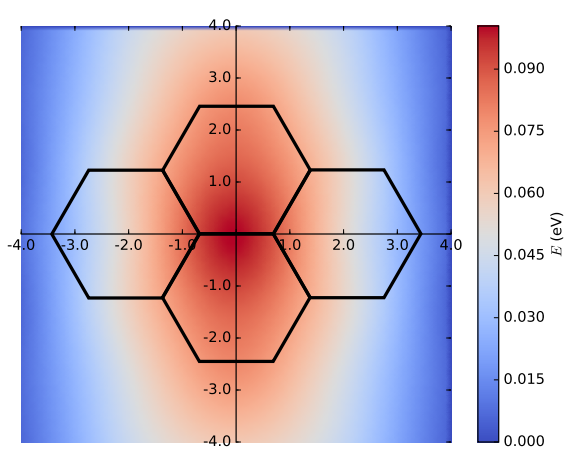

b)

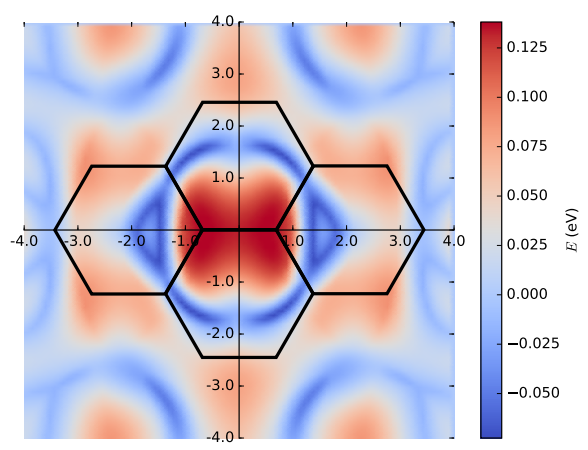

Figure 6: ( $\mathrm{a}$ and $\mathrm{b}$ ) Long- and short-range coupling for the parallel displacement of the pyrene monomers with fixed interplanar distance of $3.3 \AA$.

In order to extract the values of the long- and short-range couplings we use our ab initio calculated potential energy surfaces and calculate the long-range coupling independently by using the TrEsp procedure introduced by Madjet et al. ${ }^{73}$ Here, atom centered transition charges are fitted in order to reproduce the electrostatic potential of the electronic transition density of the monomer and the electronic coupling is calculated as:

$$
V_{L R}=\sum_{i, j} \frac{q_{e g, i}^{A} q_{e g, j}^{B}}{\left|\mathbf{R}_{i}^{A}-\mathbf{R}_{j}^{B}\right|}
$$

where the $q_{e g, i}^{A}$ and $q_{e g, j}^{B}$ denote partial transition charges at the monomer subunits $A$ and $B$ and $\mathbf{R}_{i}^{A}$ and $\mathbf{R}_{j}^{B}$ are atomic position vectors for the atoms $i$ and $j$ belonging to different monomers. For two identical monomers the total electronic coupling can be obtained from the adiabatic energies of those two excited states, which follow the diabatic character of the first two excited states at the equilibrium geometry. The total coupling can then be 
calculated as

$$
V=\frac{E_{2}-E_{1}}{2}
$$

Therefore, since the long-range part of the coupling can be independently calculated, the short-range part of the coupling can be extracted from Eq. 11. Notice, that this approach can be generalized to hetero-dimers as shown by Scholes et al. ${ }^{74}$ and Madjet et al. ${ }^{75}$

In Fig. 6 we present calculated couplings for different relative positions of the pyrene monomers within the dimer. As can be seen from Fig. 6a the parallel displacement along the $R_{x}$ and $R_{y}$ coordinates leads to a smooth variation of the long-range electronic coupling which exhibits largest value close for the perfectly stacked structure. In contrast, the short range coupling shows more complicated behavior since it changes with the orbital overlap between the two monomers which is strongly dependent on the relative position of the monomers and exhibits also sign variations (cf. Fig. 6b). The change of the sign in the short-range coupling is responsible for the change of the diabatic character of the $S_{1}$ and $S_{2}$ state at the intersection of the $S_{1}$ and $S_{2}$ states (cf. Fig. 5). In general, in dimers consisting of two identical chromophores conical intersections between the excitonic states may arise at geometries where the total coupling changes its sign and the diagonal diabatic energies become equal. Such conical intersections can lead to ultrafast electronic relaxation and their presence might have a direct influence on the functional properties of stacked molecular assemblies.

Nonadiabatic dynamics of excimer formation: Gas phase vs. crystal. In order to determine the mechanism that governs the excited state relaxation and leads to the excimer formation, we have performed trajectory surface hopping dynamics simulations in the frame of TDDFT at the BH-LYP/def2-SVP level of theory. The two lowest excited states $\left(S_{1}\right.$ and $\left.S_{2}\right)$ and the ground state have been included and the nonadiabatic couplings between all states has been calculated. Due to the high computational demand and relatively long simulation time of 2 ps we were only able to propagate a limited number of 20 trajectories at the highest BH-LYP level of theory. The results have been further confirmed by running 
more approximate lc-TDDFTB dynamics ${ }^{52,53}$ over 5 ps and using a larger ensemble of 50 trajectories. Since the two lowest excited states cross and exchange their electronic diabatic character close to the initial ground state geometry, each trajectory has been initiated in the state that is bright at the given initial geometry. The time-dependent excited state populations obtained from the nonadiabatic dynamics (cf. Fig. 7) do not exhibit simple ultrafast decay but undergo strong oscillations due to the large coupling between the $S_{1}$ and $S_{2}$ state and their almost degeneracy.

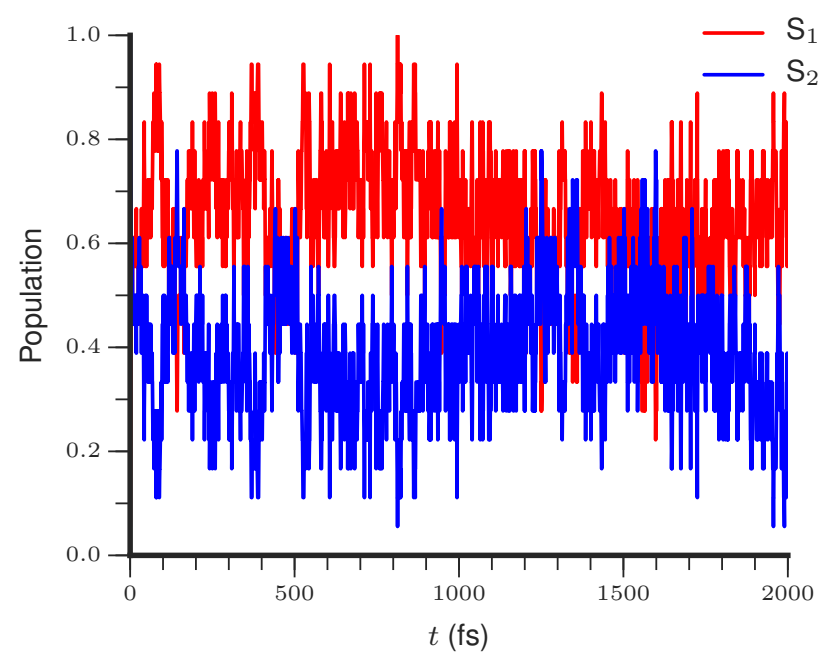

Figure 7: Time-dependent ensemble populations of the $S_{2}$ and $S_{1}$ states obtained from the surface hopping nonadiabatic dynamics simulations.

No relaxation to the electronic ground state has been observed during the simulation time, which is consistent with the observed fluorescence of pyrene dimer and with the experimental results described above. Based on the results of the nonadiabatic dynamics simulations, two distinct excimer formation channels have been identified in the gas phase: Depending on the initial energy and the geometry distortion, the trajectories either remain trapped close to the FC region and undergo vibrational dynamics mainly along the intermolecular stacking coordinate as the excimer state is formed, or they undergo large amplitude motion towards the perfectly stacked global minimum in the first excited state along the parallel shift coordinate which is accompanied by a simultaneous decrease of the perpendicular distance. 
Both relaxation channels can be qualitatively understood based on the shape of the potential energy surfaces shown in Fig. 5 that exhibit a local minimum close to the FC geometry and a global minimum at the perfectly stacked geometry. As an illustration, the excited state energies along a single trajectory that undergoes the parallel shift are presented in Fig. 8a.

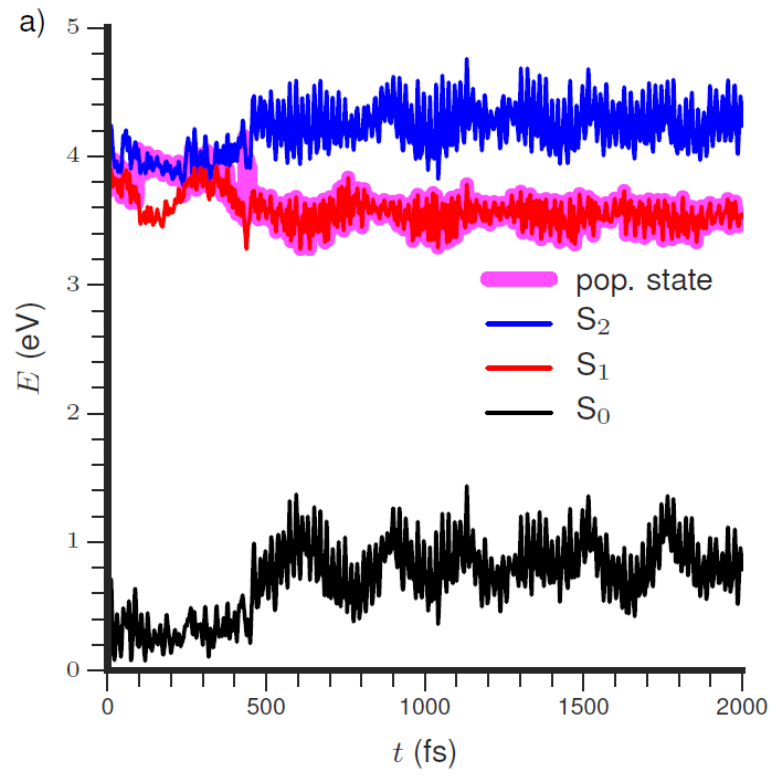

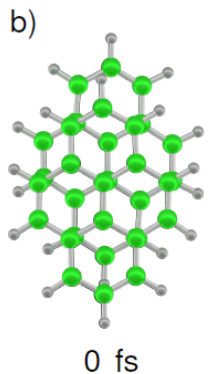

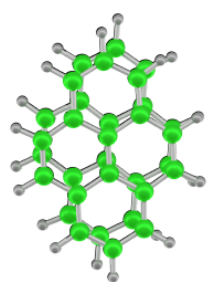

500 fs

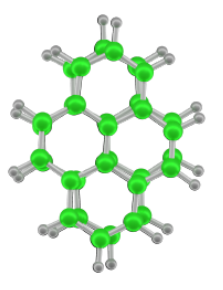

1000 fs

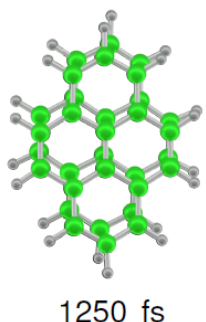

1250 fs

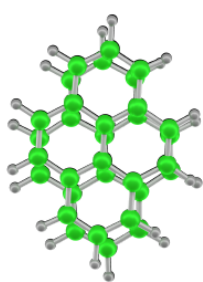

800 fs

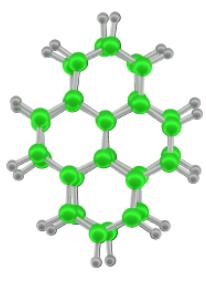

1800 fs

Figure 8: (a) Energies of the ground and two lowest excited states along a typical nonadiabatic trajectory exhibiting parallel shift towards the perfectly stacked structure. The thick magenta colored curve marks the state in which the trajectory resides. (b) Snapshots of the geometries along the same trajectory for selected times. 


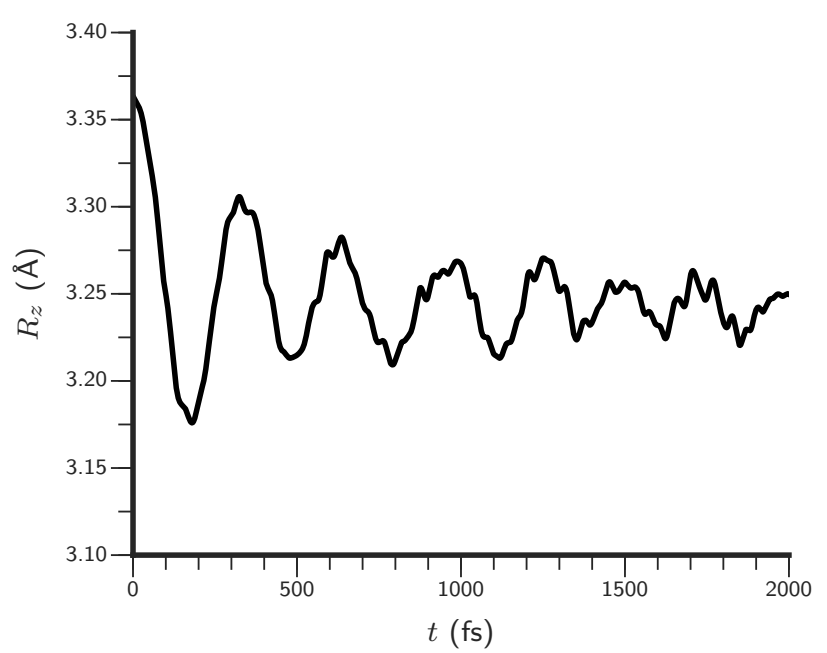

Figure 9: Interplanar distance $\left(R_{z}\right)$ between the pyrene monomers in the gas phase as a function of time averaged over an ensemble of surface hopping trajectories.

After initial excitation, the trajectory shown in Fig. 8a switches between the $S_{2}$ and $S_{1}$ state within the first 500 fs while the two pyrene monomers slide along the parallel shift coordinate as can be seen from the dynamics snapshots shown in Fig 8b. After approximately 1 ps the perfectly stacked structure is reached for the first time and the energy gap between the $S_{1}$ and $S_{2}$ opens. Since the energy is only slowly dissipated away from the parallel shift coordinate the trajectory overshoots the stacked structure and exhibits damped oscillations about the global minimum geometry which persist for several picoseconds. As already mentioned, the opening of the energy gap can be largely attributed to the increase in the short-range coupling at the stacked geometry due to the favorable orbital overlap. The other part of the trajectory ensemble (not shown), remains trapped close to the FC geometry and switches back and forth between both excited states as it passes the crossing region. In all trajectories, the perpendicular distance between the monomer units decreases with time as can be seen from Fig. 9 reflecting the stronger binding in the excited state which is a characteristic of the excimer formation.

The stabilization of the excimer requires the removal of energy from the stacking coordinate and its internal redistribution over all available vibrational modes. Thus, the excimer 
formation time is intimately connected with the rate of energy dissipation from the initially excited stacking and parallel shift coordinate into other (bath) modes. The time-scale for the internal energy redistribution can be inferred from Fig. 9 where we plot the average interplanar distance for the whole trajectory ensemble as a function of time. Initially, the distance drops from $3.36 \AA$ to $3.18 \AA$ within the first 100 fs and exhibits subsequently damped oscillations with a period of $\approx 350$ fs that persist for the whole simulation period of 2 ps. Since the full TDDFT nonadiabatic dynamics cannot be extended beyond 2 ps due to the high computational demand, we have performed more approximate lr-TDDFTB simulations over a longer time span. The lr-TDDFTB simulations (see Fig. S5) show that the oscillations fully decay on a time-scale of $\approx 5 \mathrm{ps}$ which is in a good agreement with the experimental time constant of 6-7 ps. Thus, we can conclude that the excimer stabilization in the gas-phase pyrene dimer occurs on a time-scale of several ps and is largely governed by the efficiency of the intramolecular energy redistribution. In the following we explore the influence of the crystal environment on the excimer formation in the frame of QM/MM nonadiabatic dynamics simulations on a pyrene crystal model in the frame of the lr-TDDFTB.

The excited state dynamics in the crystal environment exhibits several differences with respect to the gas phase dynamics. We present in Fig. 10 average values of the parallel shift and perpendicular distance coordinates obtained from the gas phase and crystal nonadiabatic dynamics. The gas phase results have been separated into two excimer formation channels described above (cf. full and dashed red curves in Figs. 10a and b). In contrast to the dynamics in the gas phase, the oscillation in the parallel shift coordinate is faster and more strongly damped in the crystal environment. Within the first 300 fs the parallel shift coordinate decreases rapidly from $1.2 \AA$ to about $0.6 \AA$, which is followed by a slower relaxation with the shift reaching the value of $1.0 \AA$ after 5 ps. The differences are also visible in the time-dependence of the stacking coordinate which is shown in Fig. 10b. Notice, that the stacking coordinate has generally lower value in the TDDFTB simulations due to the overestimation of the dispersion energy. The stacking coordinate exhibits oscillations that 

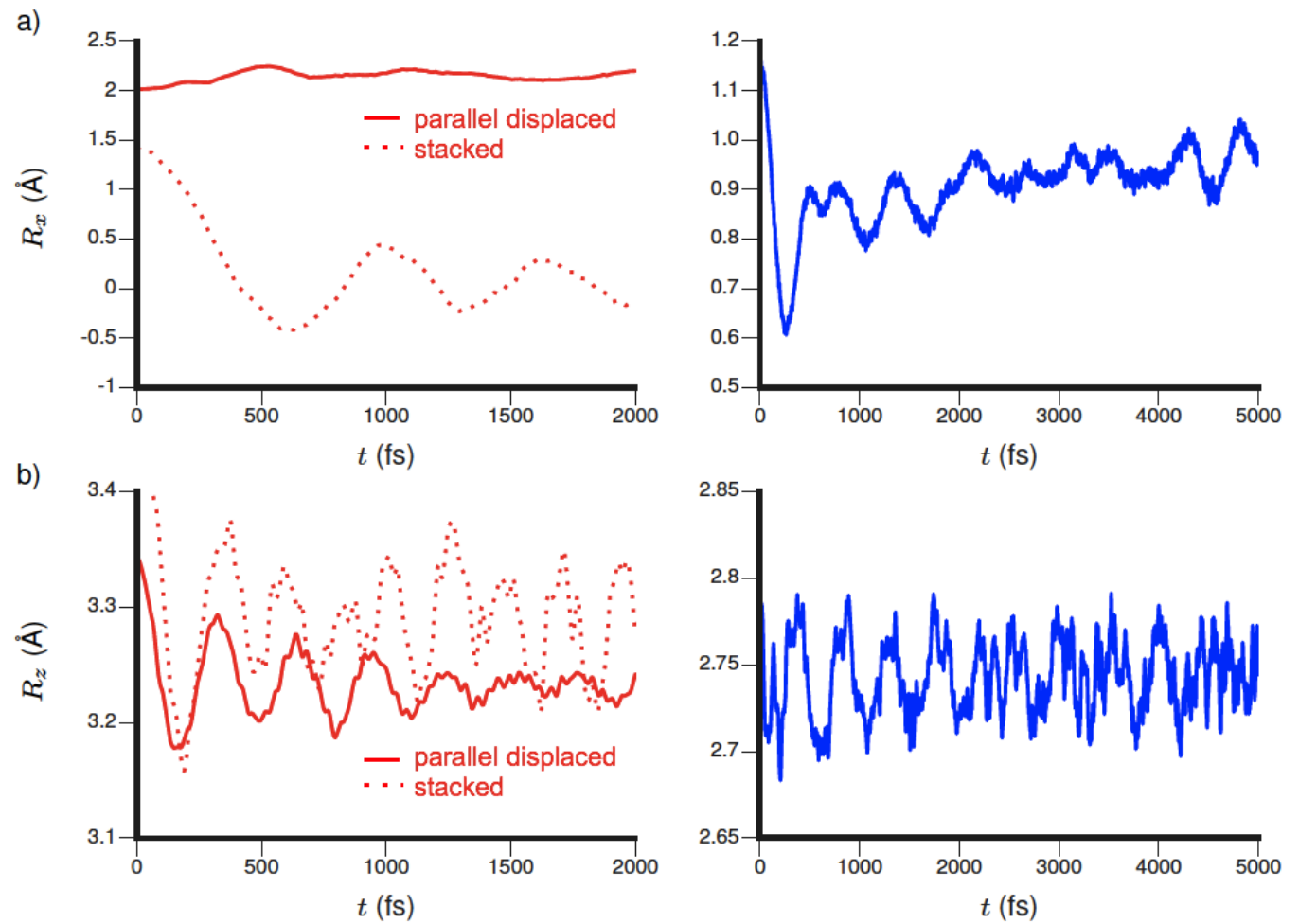

Figure 10: (a) Average value of the parallel shift coordinate $R_{x}$ (defined in the inset of Fig. 5a) as a function of time for the gas phase pyrene dimer in the frame of TDDFT (BH-LYP/def2-SVP) (red) and for pyrene dimer embedded in a crystal environment in the frame of TDDFTB (blue). The gas phase values have been divided into two excimer forming channels, with the full red line as the parallel slipped one (72\% of the trajectories) and the dashed line as the stacked pyrene excimer (28\% of the trajectories).(b) Average interplanar distance $R_{z}$ as a function of time. Labeling is the same as in part a). 
persist over the whole period of 5 ps and are less damped than in the gas phase case. This is due to the absence of the second excimer formation channel that involves significant parallel shift and leads to stronger damping in the gas phase simulation. In fact, when compared to the gas phase relaxation channel that does not involve parallel shift (dashed red line in Fig. 10) the period of the oscillation and damping are similar both in the gas phase and in the crystal. As an illustration of the crystal dynamics we present in Fig. 11a structures sampled from a single nonadiabatic trajectory over the time interval of 5 ps as well as the electronic state energies as a function of time in Fig. 11b. After initial excitation of the $S_{1}$ exciton state, a fast opening of the energy gap between the excited states is observed within the first 300 fs that accompanies the motion along the parallel shift coordinate. After the collision with the surrounding environment, the gap becomes again smaller and continues to fluctuate over the whole simulation period.
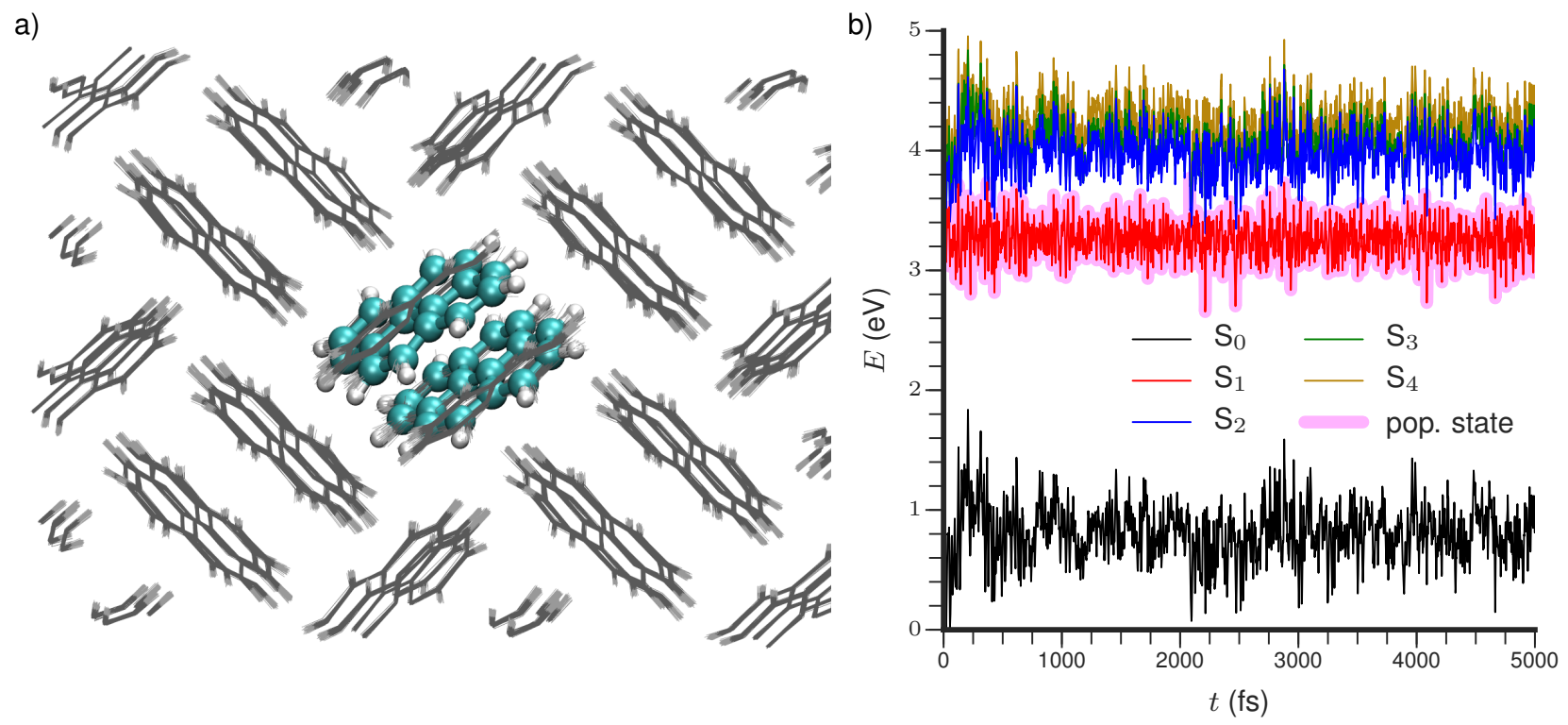

Figure 11: (a) Section from the QM/MM model for nonadiabatic dynamics of pyrene dimer in a crystal. In the figure structures from a single nonadiabatic trajectory have been superposed. (b) Ground and excited state energies of pyrene dimer (QM part of the model) as a function of time. The thick magenta line labels the occupied state during the surface hopping simulation. 


\section{Conclusion}

We carried out a joint experimental and theoretical study of the ultrafast excimer formation dynamics in pyrene dimer, arguably the most important model for excimer photophysics in general. The nonadiabatic dynamics was simulated in the frame of TDDFT and combined with time-resolved picosecond ionization experiments. The simulations in the gas phase led to the identification of two excimer formation channels involving: (i) local relaxation along the stacking coordinated close to the FC window and (ii) large amplitude oscillations along the parallel shift coordinate accompanied by damped oscillations along the stacking direction. Both processes lead to the stabilization of the excimer on a time scale of several ps. The excited state dynamics is characterized by a strong coupling between the two lowest exciton states due to the presence of conical intersections between the delocalized excitonic states. Such conical intersections arise due to the interplay between the long-range and shortrange electronic coupling and may play an important role in the excited state dynamics in materials containing stacked $\pi$-conjugated systems. The simulations were supported by the picosecond photoionization experiments in a supersonic jet that reveal excited state dynamics with a time constant of 6-7 ps that is assigned to excimer formation. Experimental and computed time constants are thus in good agreement. The redistribution of vibrational energy in the excited state upon excimer formation leads to a change in the ionization

probability that manifests itself in a transient contribution to the ion signal. Experiments were conducted at several excitation wavelength and dissociative photoionization of larger clusters was successfully suppressed in most experiments.

In addition to the experimental and theoretical study of the gas phase dynamics we also explored the influence of the crystal environment on the excimer formation with the help of QM/MM lc-TDDFTB nonadiabatic dynamics simulations on a crystal model. The excimer formation in the crystal environment proceeds along a single pathway that involves strongly damped motion along the parallel shift coordinate accompanied by oscillations along the stacking direction decaying on a time scale larger than 5 ps. Our findings provide 
a first detailed picture of the excimer formation dynamics starting from a van der Waals weakly bound dimer with preoriented pyrene units and show that dynamical effects should play an important role in the design of novel organic functional materials. The function

of even perfectly structurally designed molecular material may rapidly deteriorate due to the ultrafast excimer formation mediated by the efficient nonradiative processes at conical intersections. Our findings may have implications for the exciton transfer in molecular crystals which can vary between the incoherent hopping and coherent transport mediated by dynamic delocalization. ${ }^{76}$

\section{Acknowledgement}

JH, AH, RM and MISR gratefully acknowledge the financial support by the European Research Council (ERC) Consolidator grant DYNAMO (Grant Nr. 646737). IF would like to thank the German Science Foundation, DFG for funding via project FI575/9-x. HCS acknowledges a fellowship by the Fonds der Chemischen Industrie.

\section{References}

(1) Förster, T. Angew. Chem. Int. Ed. 1969, 8, 333-343.

(2) Klopffer, W.; Birks, J. Organic Molecular Photophysics; 1973; pp 357-402.

(3) Lim, J. M.; Kim, P.; Yoon, M.-C.; Sung, J.; Dehm, V.; Chen, Z.; Würthner, F.; Kim, D. Chem. Sci. 2013, 4, 388-397.

(4) Marciniak, H.; Li, X.-Q.; Würthner, F.; Lochbrunner, S. J. Phys. Chem. A 2011, 115, 648.

(5) Ye, T.; Singh, R.; Butt, H.-J.; Floudas, G.; Keivanidis, P. E. ACS Appl. Mater. Interfaces 2013, 5, 11844. 
(6) Chen, Z.; Stepanenko, V.; Dehm, V.; Prins, P.; Siebbeles, L. D. A.; Seibt, J.; Marquetand, P.; Engel, V.; Würthner, F. Chem. Eur. J. 2007, 13, 436.

(7) Howard, I. A.; Laquai, F. D. R.; Keivanidis, P. E.; Friend, R. H.; Greenham, N. C. J. Phys. Chem. C 2009, 113, 21225.

(8) Cook, R. E.; Phelan, B. T.; Kamire, R. J.; Majewski, M. B.; Young, R. M.; Wasielewski, M. R. J. Phys. Chem. A 2017, 121, 1607-1615.

(9) Smith, M. B.; Michl, J. Annu. Rev. Phys. Chem. 2013, 64, 361-386.

(10) Mauck, C. M.; Hartnett, P. E.; Margulies, E. A.; Ma, L.; Miller, C. E.; Schatz, G. C.; Marks, T. J.; Wasielewski, M. R. J. Am. Chem. Soc. 2016, 138, 11749-11761.

(11) Beljonne, D.; Yamagata, H.; Brédas, J. L.; Spano, F. C.; Olivier, Y. Phys. Rev. Lett. 2013, 110, 226402.

(12) Walker, B. J.; Musser, A. J.; Beljonne, D.; Friend, R. H. Nat. Chem. 2013, 5, 1019.

(13) Eaton, S. W.; Shoer, L. E.; Karlen, S. D.; Dyar, S. M.; Margulies, E. A.; Veldkamp, B. S.; Ramanan, C.; Hartzler, D. A.; Savikhin, S.; Marks, T. J. J. Am. Chem. Soc. 2013, 135, 14701.

(14) Miller, C. E.; Wasielewski, M. R.; Schatz, G. C. J. Phys. Chem. C 2017, 121, 1034510350.

(15) Chen, S.; Wang, L.; Fahmi, N. E.; Benkovic, S. J.; Hecht, S. M. J. Am. Chem. Soc. 2012, 134, 18883-18885.

(16) Santiago-Gonzalez, B.; Monguzzi, A.; Azpiroz, J. M.; Prato, M.; Erratico, S.; Campione, M.; Lorenzi, R.; Pedrini, J.; Santambrogio, C.; Torrente, Y. Science 2016, 353, 571-575. 
(17) Jumper, C. C.; Anna, J. M.; Stradomska, A.; Schins, J.; Myahkostupov, M.; Prusakova, V.; Oblinsky, D. G.; Castellano, F. N.; Knoester, J.; Scholes, G. D. Chem. Phys. Lett. 2014, 599, 23-33.

(18) Musser, A. J.; Liebel, M.; Schnedermann, C.; Wende, T.; Kehoe, T. B.; Rao, A.; Kukura, P. Nat. Phys. 2015, 11, 352-357.

(19) Tamura, H. J. Phys. Chem. A 2016, 120, 93419347.

(20) Förster, T.; Kasper, K. Berichte der Bunsengesellschaft für physikalische Chemie 1955, 59, 976-980.

(21) Saigusa, H.; Lim, E. C. Acc. Chem. Res. 1996, 29, 171-178.

(22) Miyasaka, H.; Masuhara, H.; Mataga, N. J. Phys. Chem. 1985, 89, 1631-1636.

(23) Saigusa, H.; Lim, E. C. Chem. Phys. Lett. 2001, 336, 65-70.

(24) Willberg, D.; Gutmann, M.; Breen, J.; Zewail, A. J. Chem. Phys. 1992, 96, 198-212.

(25) Hertel, I.; Radloff, W. Rep. Prog. Phys. 2006, 69, 1897.

(26) Knochenmuss, R.; Fischer, I.; Lührs, D.; Lin, Q. Isr. J. Chem. 1999, 39, 221-230.

(27) Lührs, D. C.; Knochenmuss, R.; Fischer, I. Phys. Chem. Chem. Phys. 2000, 2, 43354340 .

(28) Piuzzi, F.; Dimicoli, I.; Mons, M.; Millié, P.; Brenner, V.; Zhao, Q.; Soep, B.; Tramer, A. Chem. Phys. 2002, 275, 123-147.

(29) Miyazaki, M.; Fujii, M. Phys. Chem. Chem. Phys. 2015, 17, 25989-25997.

(30) Birks, J. Rep. Prog. Phys. 1975, 38, 903.

(31) Turro, N. J.; Ramamurthy, V.; Scaiano, J. C. Modern Molecular Photochemistry of Organic Molecules; 2010. 
(32) Andriessen, R.; Ameloot, M.; Boens, N.; De Schryver, F. C. J. Phys. Chem. 1992, 96, $314-326$.

(33) Iyer, E. S. S.; Sadybekov, A.; Lioubashevski, O.; Krylov, A. I.; Ruhman, S. J. Phys. Chem. A 2017, 121, 1962-1975.

(34) Becke, A. Phys. Rev. A 1988, 38, 3098.

(35) Lee, C.; Yang, W.; Parr, G. R. Phys. Rev. B. 1988, 37, 785.

(36) Becke, A. D. J. Chem. Phys. 1993, 98, 1372.

(37) TURBOMOLE V6.3 2010, a development of University of Karlsruhe and Forschungszentrum Karlsruhe GmbH, 1989-2007, TURBOMOLE GmbH, since 2007; available from http://www. turbomole.com.

(38) Yanai, T.; Tew, D. P.; Handy, N. C. Chem. Phys. Lett. 2004, 393, 51-57.

(39) Frisch, M. J.; Trucks, G. W.; Schlegel, H. B.; et. al., Gaussian 09, Rev. A.02, Gaussian Inc., Wallingford, CT; 2009.

(40) Schäfer, A.; Huber, C.; Ahlrichs, R. J. Chem. Phys. 1994, 100, 2975.

(41) Weigend, F.; Häser, M.; Patzelt, H.; Ahlrichs, R. Chem. Phys. Lett. 1988, 294, 143.

(42) Grimme, S.; Antony, J.; Ehrlich, S.; Krieg, H. J. Chem. Phys. 2010, 132, 154104.

(43) Huenerbein, R.; Grimme, S. Chem. Phys. 2008, 343, 362.

(44) Tully, J. C. J. Chem. Phys. 1990, 93, 1061.

(45) Werner, U.; Mitrić, R.; Bonačić-Koutecký, V. Chem. Phys. 2008, 349, 319.

(46) Mitrić, R.; Werner, U.; Bonačić-Koutecký, V. J. Chem. Phys. 2008, 129, 164118. 
(47) Mitrić, R.; Werner, U.; Wohlgemuth, M.; Seifert, G.; Bonačić-Koutecký, V. J. Phys. Chem. A 2009, 113, 12700.

(48) Lisinetskaya, P. G.; Mitric, R. Phys. Rev. A 2011, 83, 033408.

(49) Berendsen, H. J. C.; Postma, J. P. M.; van Gunsteren, W. F.; DiNola, A.; Haak, J. R. J. Chem. Phys. 1984, 81, 3684-3690.

(50) Werner, U.; Mitrić, R.; Bonačić-Koutecký, V. J. Chem. Phys. 2010, 132, 174301.

(51) DFTBaby - Software for lc-TD-DFT(B) calculations and non-adiabatic dynamics simulations, http://www.dftbaby.chemie.uni-wuerzburg.de,.

(52) Humeniuk, A.; Mitrić, R. J. Chem. Phys. 2015, 143, 134120.

(53) Humeniuk, A.; Mitrić, R. https://arxiv.org/abs/1703.04049 2017,

(54) Grimme, S. J. Comput. Chem. 2006, 27, 1787-1793.

(55) Chung, L. W.; Sameera, W. M. C.; Ramozzi, R.; Page, A. J.; Hatanaka, M.; Petrova, G. P.; Harris, T. V.; Li, X.; Ke, Z.; Liu, F.; Li, H.-B.; Ding, L.; Morokuma, K. Chem. Rev. 2015, 115, 5678-5796.

(56) Camerman, A.; Trotter, J. Acta Crystallogr. 1965, 18, 636-643.

(57) Mayo, S.; Olafson, B.; Goddard III, W. J. Phys. Chem. 1990, 94, 8897.

(58) Schon, C.; Roth, W.; Fischer, I.; Pfister, J.; Kaiser, C.; Fink, R. F.; Engels, B. Phys. Chem. Chem. Phys. 2010, 12, 9339.

(59) Auerswald, J.; Engels, B.; Fischer, I.; Gerbich, T.; Herterich, J.; Krueger, A.; Lang, M.; Schmitt, H.-C.; Schon, C.; Walter, C. Phys. Chem. Chem. Phys. 2013, 15, 8151.

(60) Mangle, E. A.; Topp, M. R. J. Phys. Chem. 1986, 90, 802-807.

(61) Numata, Y.; Nirasawa, T.; Suzuka, I. J. Photochem. Photobiol. A 2010, 209, 27-31. 
(62) Ohta, N.; Baba, H.; Marconi, G. Chem. Phys. Lett. 1987, 133, 222-229.

(63) Zhang, J.; Han, F.; Kong, W. J. Phys. Chem. A 2010, 114, 11117-11124.

(64) Joblin, C.; Dontot, L.; Garcia, G.; Spiegelman, F.; Rapacioli, M.; Nahon, L.; Parneix, P.; Pino, T.; Brechignac, P. http://arxiv.org/abs/1704.05683 2017,

(65) Joblin, C. personal communication.

(66) Patterson, F.; Lee, H.; Wilson, W. L.; Fayer, M. Chem. Phys. 1984, 84, 51-60.

(67) Cheng, K. A. W. Y.; Schepp, N. P.; Cozens, F. L. J. Phys. Chem. A 2004, 108, 71327134 .

(68) Costa, T.; Seixas de Melo, J. S.; Castro, C. S.; Gago, S.; Pillinger, M.; Goncalves, I. S. J. Phys. Chem. B 2010, 114, 12439.

(69) Shirai, S.; Iwata, S.; Tani, T.; Inagaki, S. J. Phys. Chem. A 2011, 115, 7687-7699.

(70) Hsu, C. P.; You, Z. Q.; Chen, H. C. J. Phys. Chem. C 2008, 112, 1204.

(71) Arago, J.; Troisi, A. Phys. Rev. Lett. 2015, 114, 026402.

(72) Koutecký, J.; Paldus, J. Theoret. Chim. Acta 1963, 1, 268-281.

(73) Madjet, M. A. E.; Abdurahman, A.; Renger, T. J. Phys. Chem. B 2006, 110, 17268.

(74) Scholes, G. D.; Gould, I.; Cogdell, R.; Fleming, G. R. J. Phys. Chem. B 1999, 110, 17268.

(75) Madjet, M. E. A.; Müh, F.; Renger, T. J. Phys. Chem. B 2009, 113, 12603.

(76) Wang, T.; Chan, W.-L. J. Phys. Chem. Lett. 2014, 5, 1812-1818. 
Graphical TOC Entry

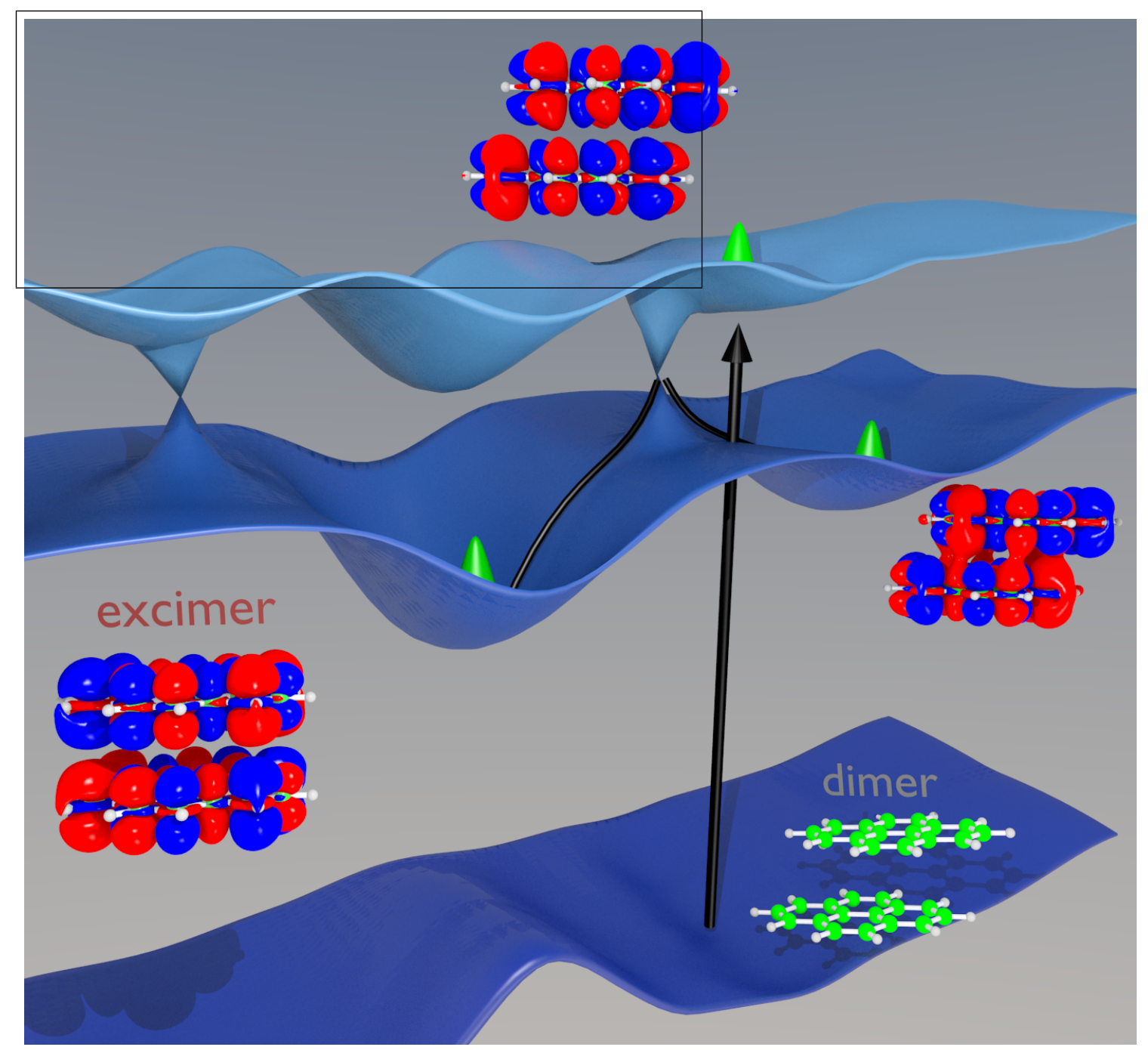




\section{The Mechanism of Excimer Formation: An Experimental and Theoretical Study on the Pyrene Dimer: Supplementary Ma- terial}

Joscha Hoche, ${ }^{a, b}$ Hans-Christian Schmitt, ${ }^{a, b}$ Alexander Humeniuk, ${ }^{a}$ Ingo Fischer ${ }^{* a}$, Roland Mitrić ${ }^{a}$ and Merle I. S. Röhr*a

\footnotetext{
${ }^{a}$ Institut für physikalische und theoretische Chemie, Julius-Maximilians-Universität Würzburg, D-97074, Würzburg, Germany E-mail: ingo.fischer@uni-wuerzburg.de,merle.roehr@uni-wuerzburg.de

$b$ These authors contributed equally to the work.
} 


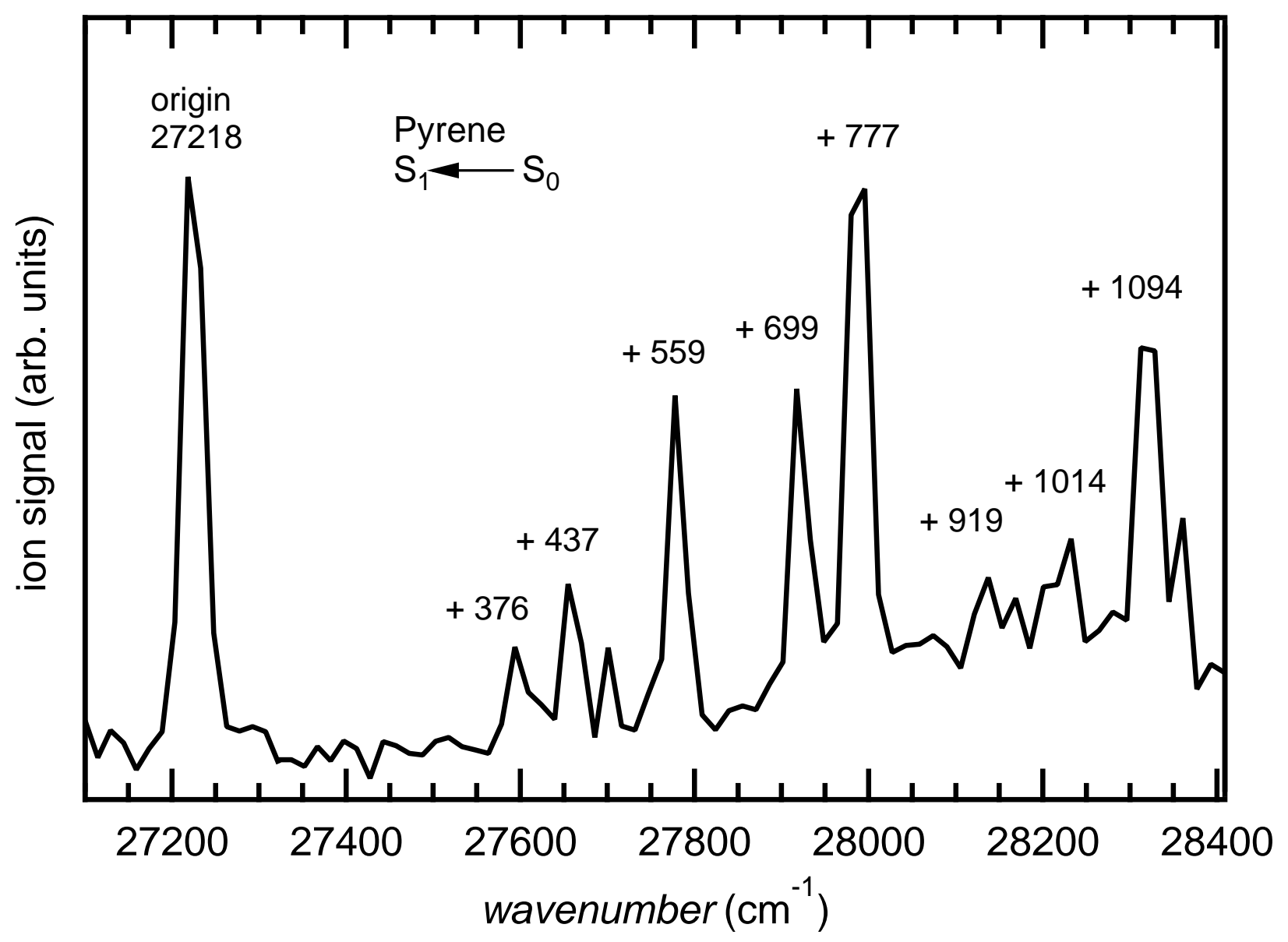

Fig. S1: $\left[1+2^{\prime}\right]$-REMPI of the $S_{1}$ state of pyrene using $527 \mathrm{~nm}$ as the probe pulses. The origin was found to be $27218 \mathrm{~cm}^{-1}$. Several well-resolved vibronic transitions are visible which indicate sufficient cooling in the molecular beam. 


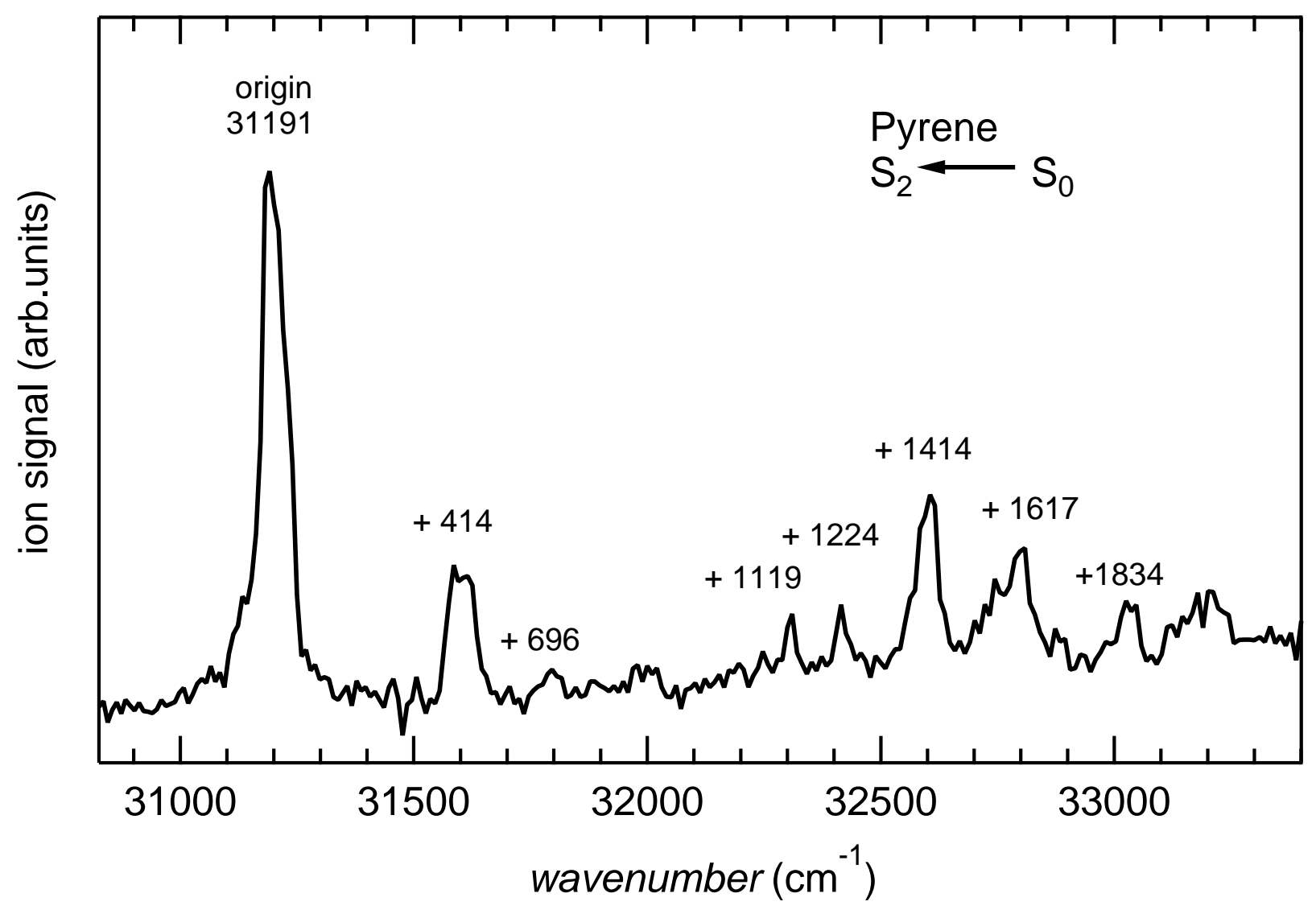

Fig. S2: $\left[1+2^{\prime}\right]$-REMPI of the $S_{2}$ state of pyrene using $351 \mathrm{~nm}$ as the probe pulses. The origin was found to be $31191 \mathrm{~cm}^{-1}$. Again several vibronic transitions are visible. 


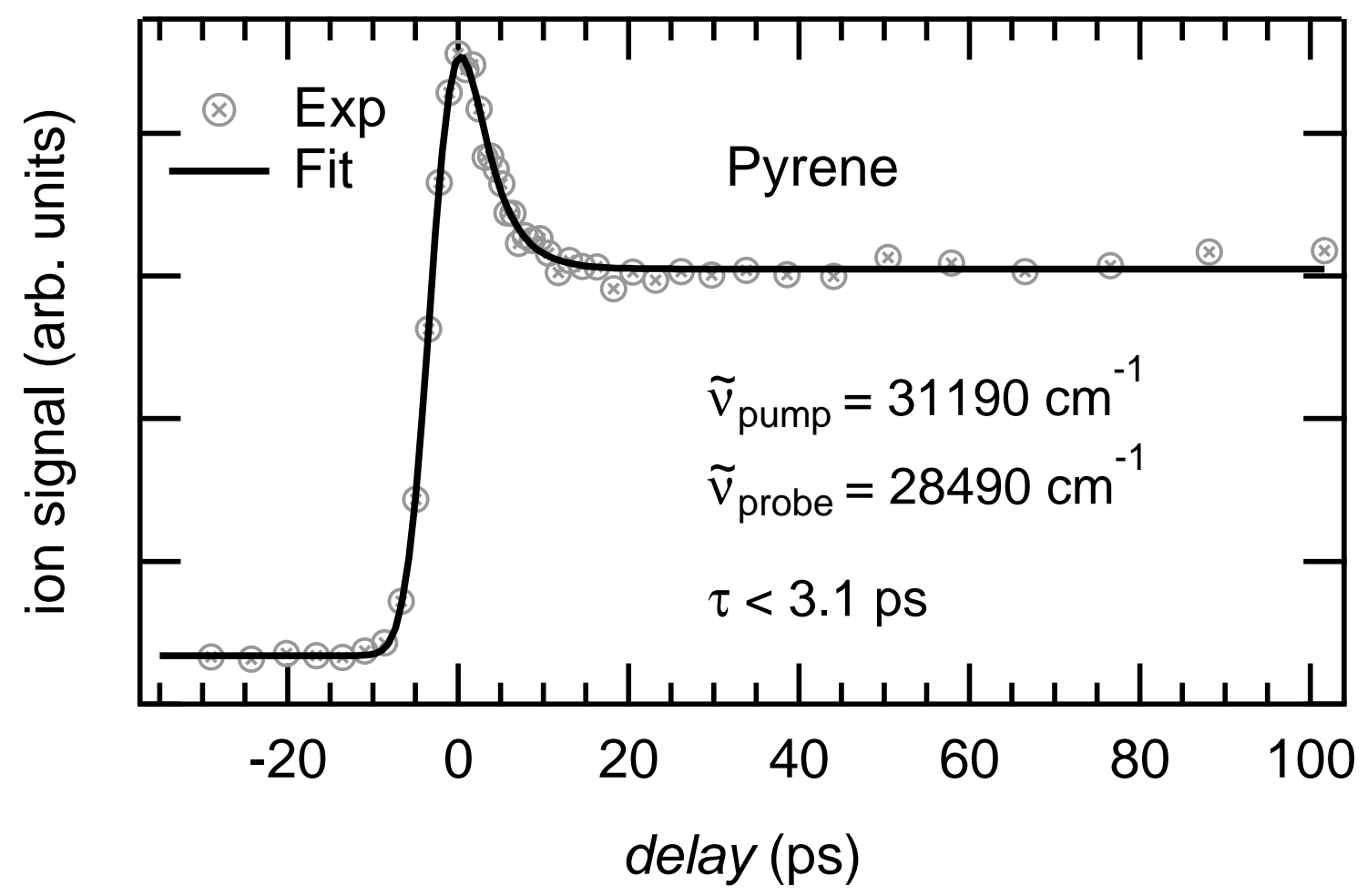

Fig. S3: Delay scan of the pyrene Monomer obtained at the origin (320.6 nm) of the $S_{2}$ state using $351 \mathrm{~nm}$ as the probe pulses. The signal decays rapidly $(<3.1 \mathrm{ps})$ to a constant level due to IVR from the $S_{2}$ zero order state to the coupled $S_{1}$ levels. 

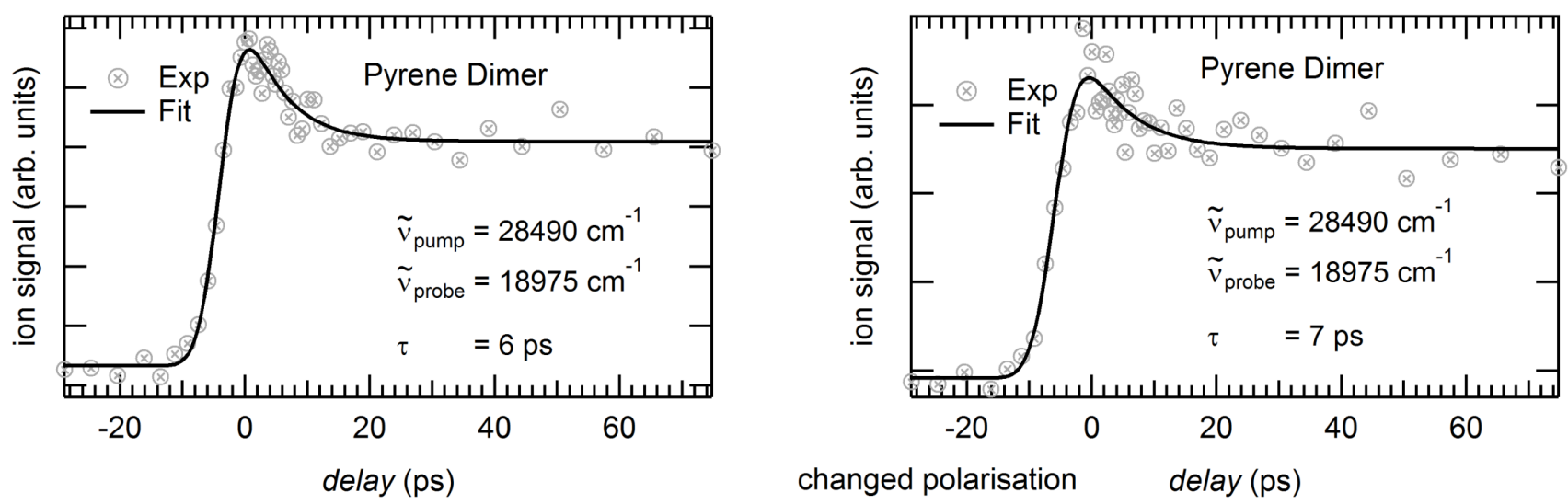

Fig. S4: Delay scans of the pyrene Dimer obtained at $351 \mathrm{~nm}$ excitation. The two spectra recorded with parallel (left) and perpendicular (right) polarization of pump- and probe $(527 \mathrm{~nm})$-laser show very similar lifetimes. This indicates that the observed transient is not related to molecular rotation. 


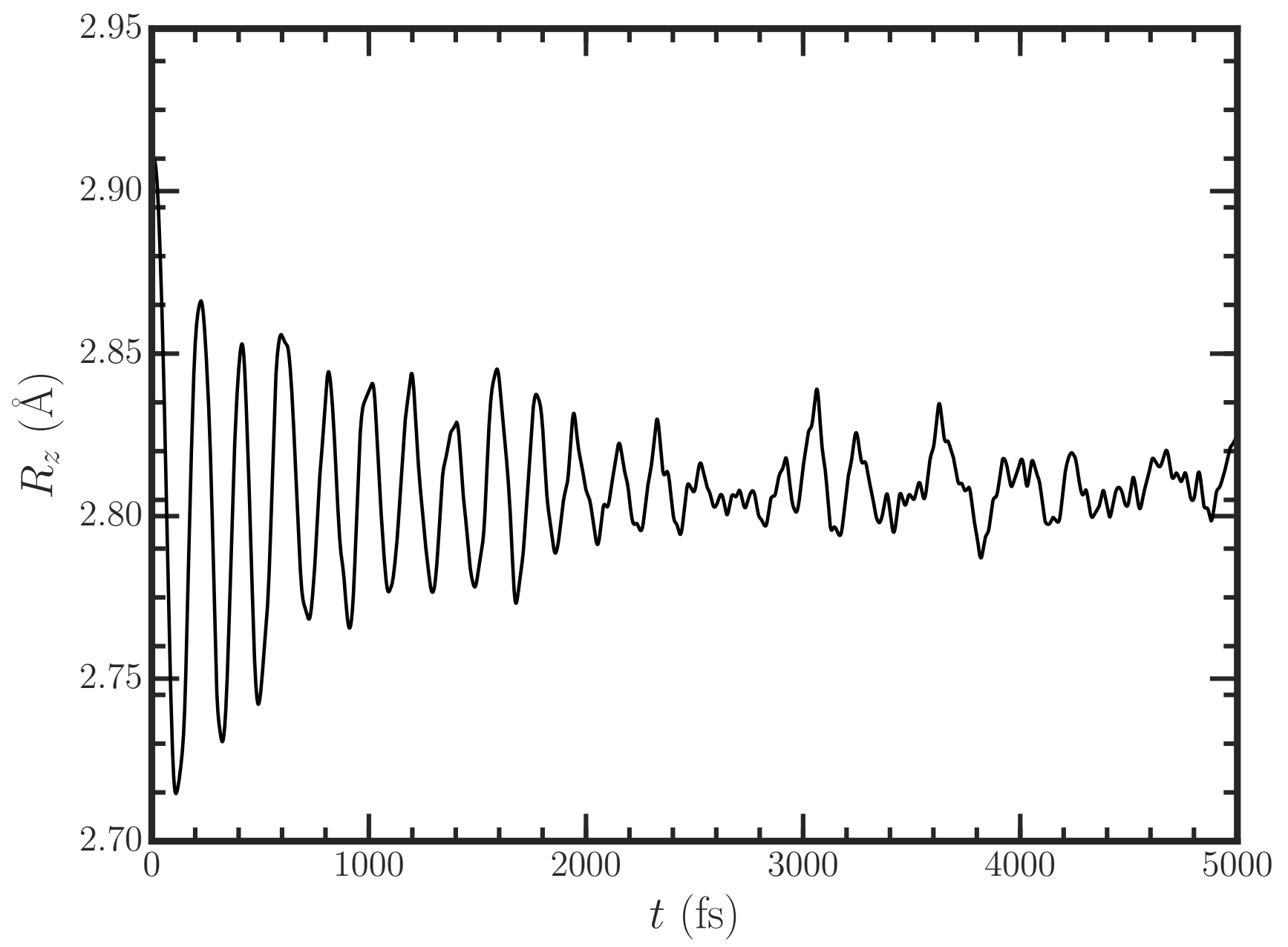

Fig. S5: Stacking distance between the pyrene monomers as a function of time averaged over an ensemble of surface hopping trajectories. 


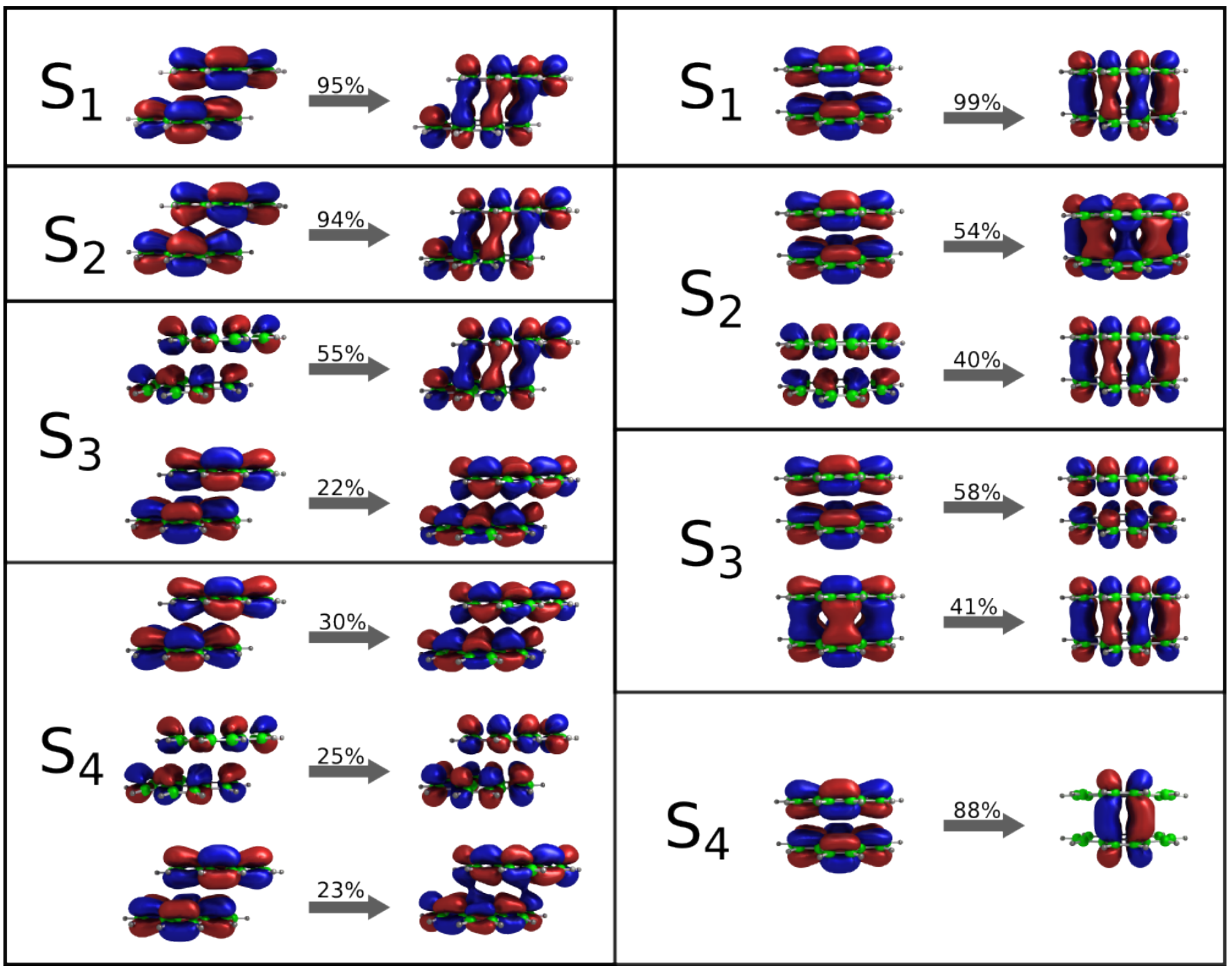

Fig. S6: Natural transition orbitals of the parallel shifted ground state geometry (left) and the fully stacked excimer structrure (right) in the framework of TDDFT CAM-B3LYP/def-2SVP. 


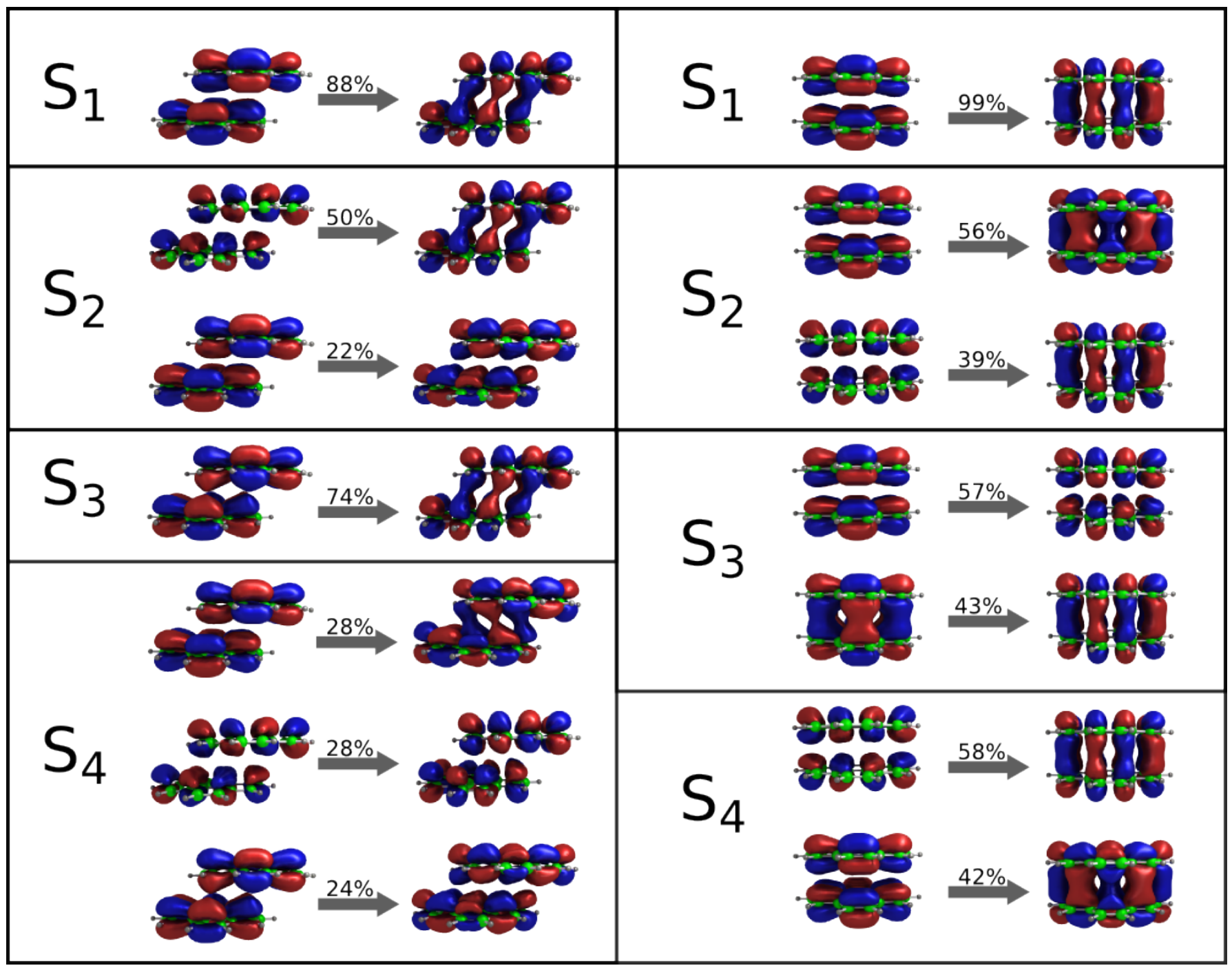

Fig. S7: Natural transition orbitals of the parallel shifted ground state geometry (left) and the fully stacked excimer structrure (right) in the framework of TDDFT BHLYP/def2-SVP. 

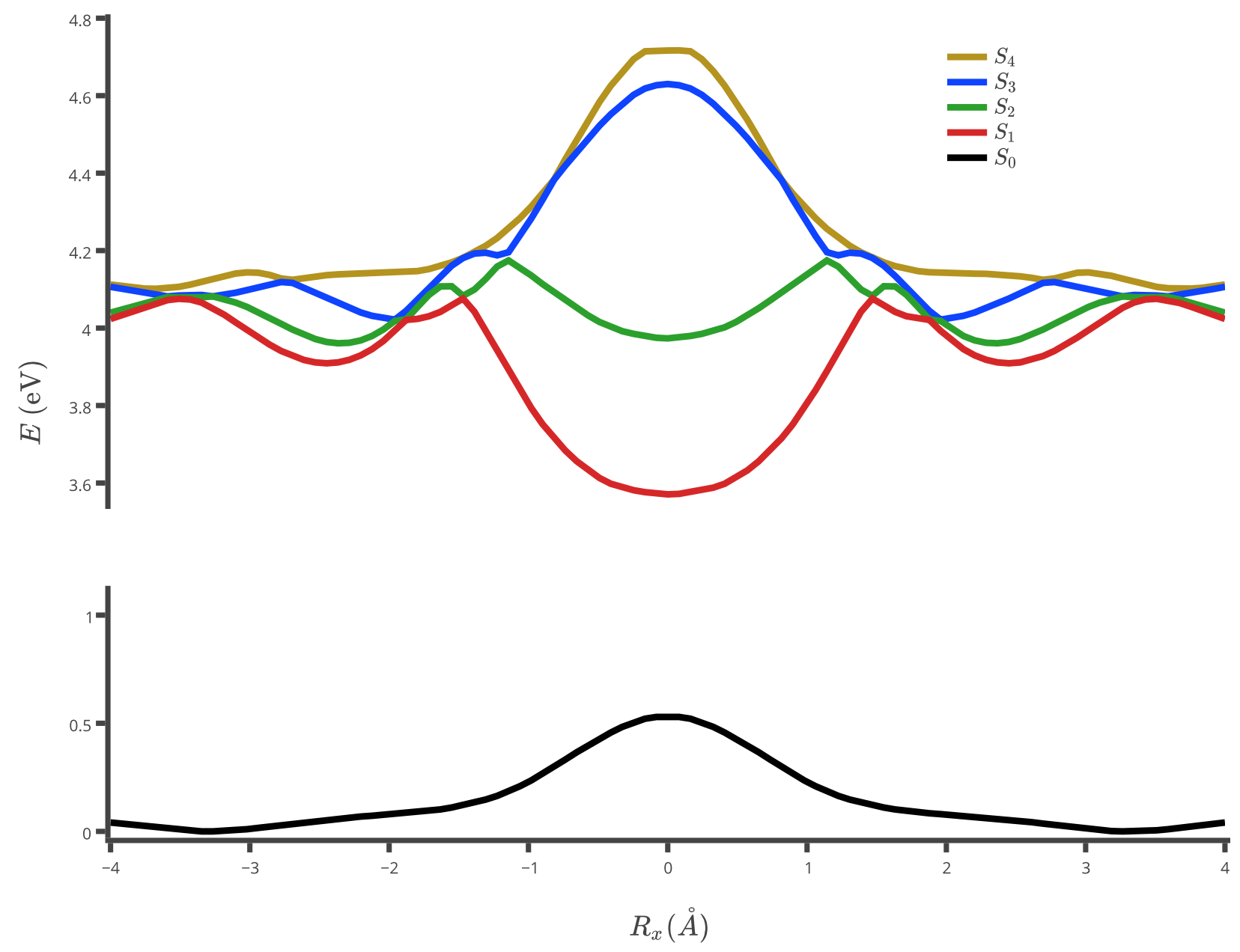

Fig. S8: Energies of the ground $\left(S_{0}\right)$ and the four lowest $\left(S_{1}-S_{4}\right)$ excited states of the pyrene dimer along the parallel shift coordinate $\left(R_{x}\right)$ at an interplanar distance $\left(R_{z}\right)$ of $3.3 \AA$. (CAM-B3LYP/def2-SVP) 


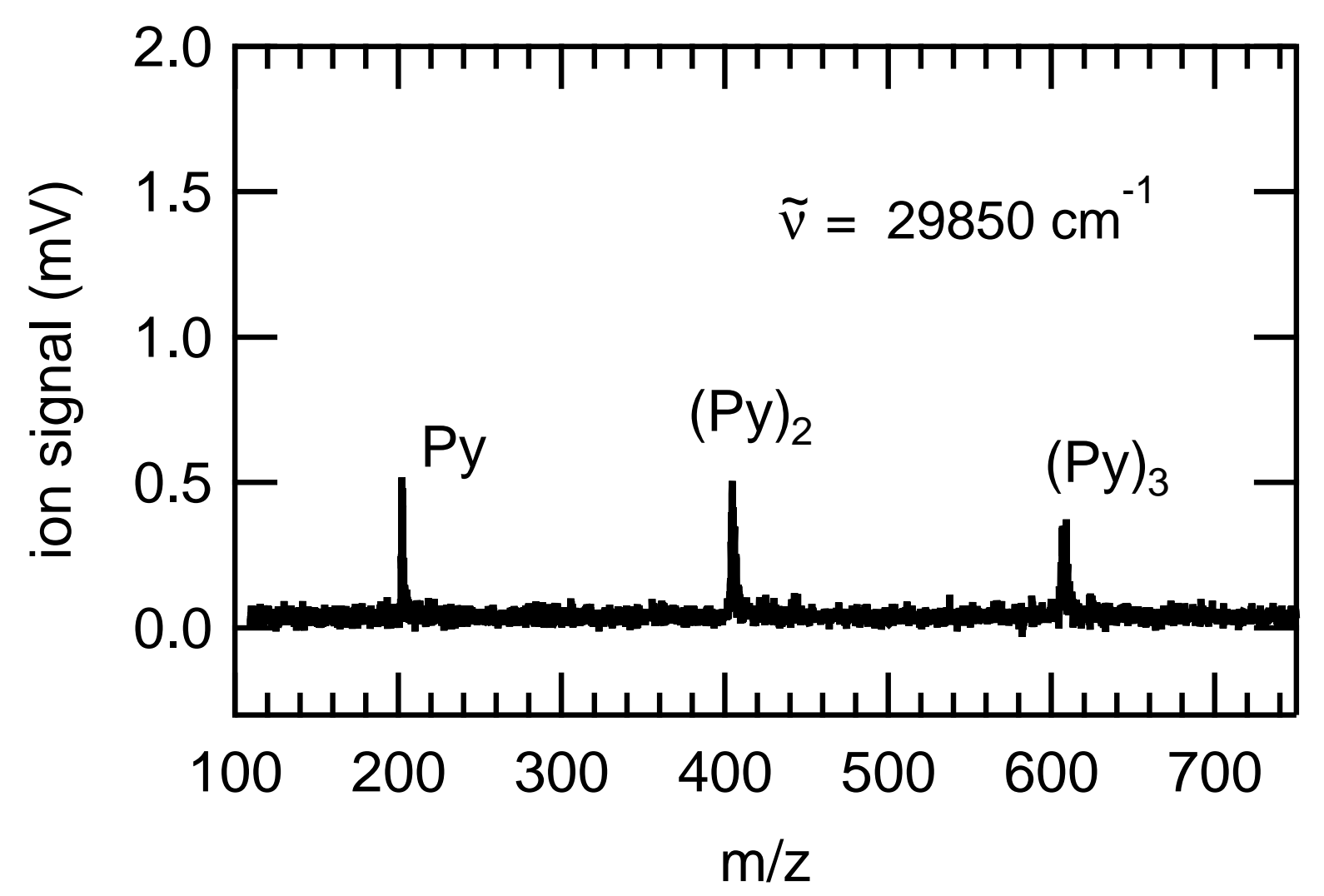

Fig. S9: Mass spectra measured at $335 \mathrm{~nm}$, which corresponds to band maximum of the dimer absorption. 


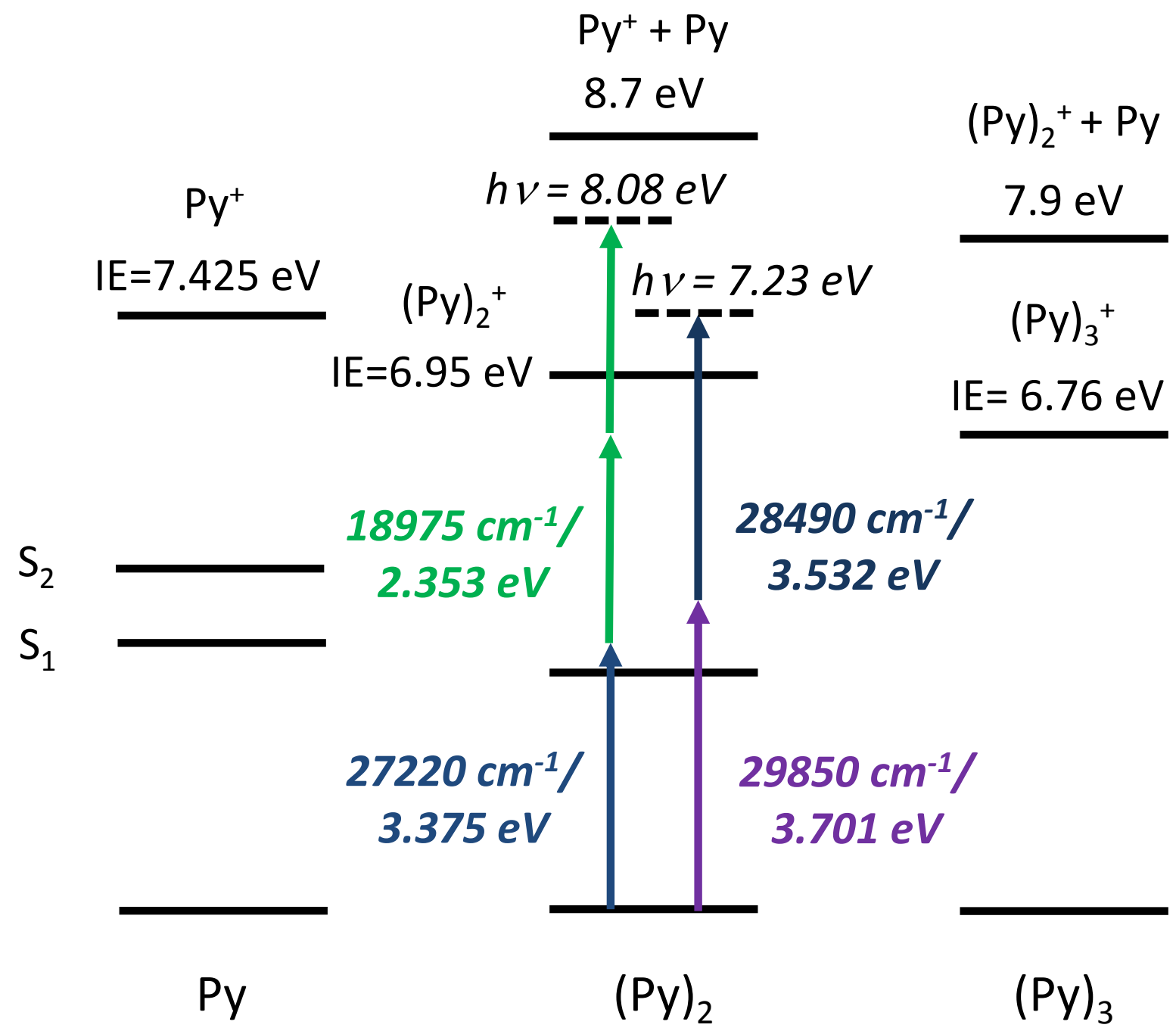

Fig. S10: Ionization and fragmentation thresholds of the pyrene monomer, dimer and trimer. 


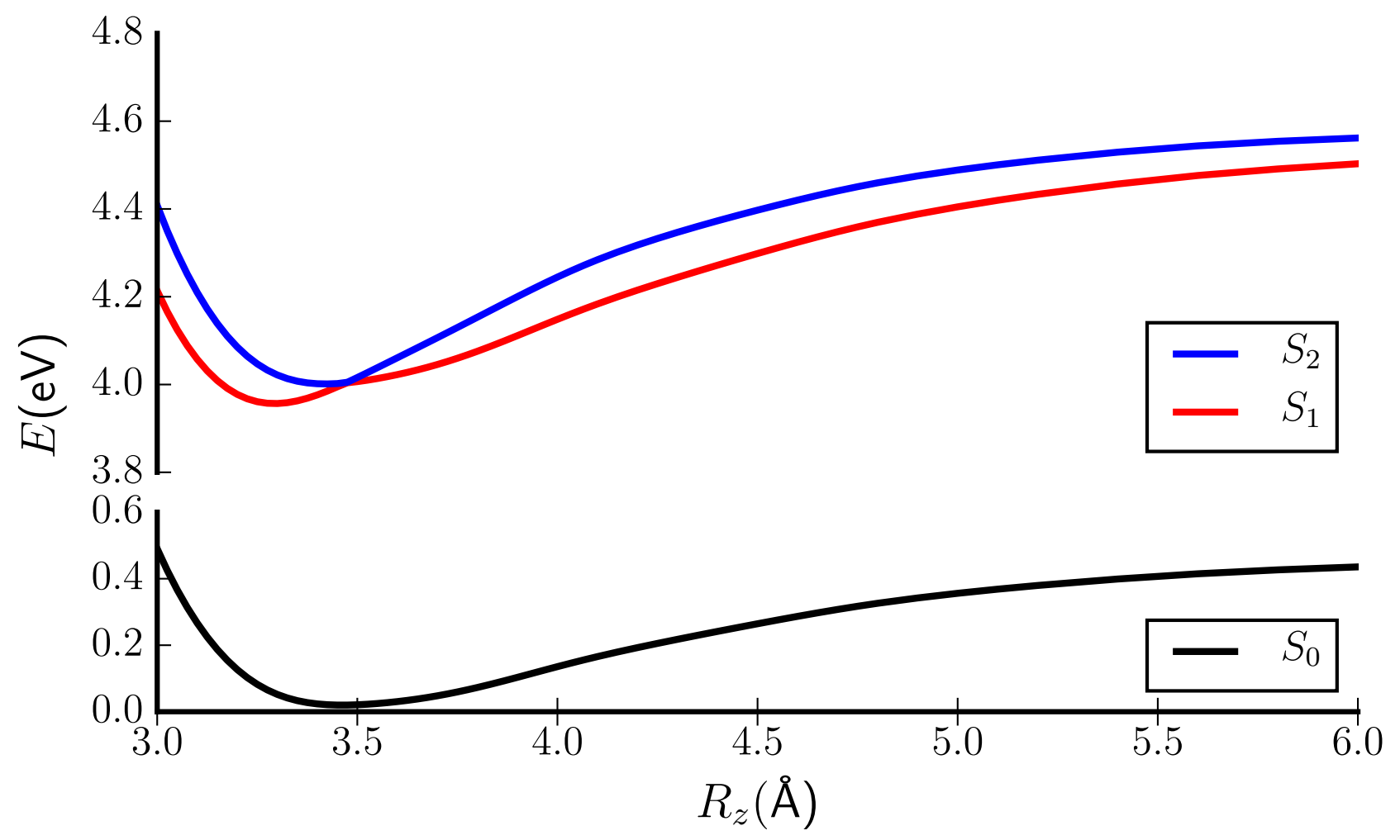

Fig. S11: Energies of the ground $\left(S_{0}\right)$ and the two lowest $\left(S_{1}, S_{2}\right)$ excited states of the pyrene dimer along the interplanar distance $\left(R_{z}\right)$ in the frame of TDDFT (BH-LYP/TZVP). 
Table S1: Calculated vertical transition energies of the ground state minimum of the pyrene dimer and monomer.

\begin{tabular}{|c|c|c|c|}
\hline & Dimer $\mathrm{S}_{1}(\mathrm{eV})$ & Dimer $\mathrm{S}_{2}(\mathrm{eV})$ & Monomer AE $(\mathrm{eV})$ \\
\hline $\begin{array}{l}\text { TDDFT } \\
\text { BH-LYP }{ }^{a}\end{array}$ & & & \\
\hline $\begin{array}{l}\text { def2-SVP } \\
6-31 \mathrm{G}^{*} \\
\text { TZVP }\end{array}$ & $\begin{array}{l}3.95 \\
4.01 \\
3.99\end{array}$ & $\begin{array}{l}3.98 \\
4.04 \\
3.99\end{array}$ & $\begin{array}{l}4.09 \\
4.14 \\
4.10\end{array}$ \\
\hline $\begin{array}{l}\text { TDDFT } \\
\text { CAM-B3LYP }^{a}\end{array}$ & & & \\
\hline $\begin{array}{l}\text { def2-SVP } \\
\text { TZVP }\end{array}$ & $\begin{array}{l}3.51 \\
3.57\end{array}$ & $\begin{array}{l}3.73 \\
3.82\end{array}$ & $\begin{array}{l}4.07 \\
4.08\end{array}$ \\
\hline TDDFTB $^{a}$ & 3.50 & 3.59 & 3.53 \\
\hline $\operatorname{MCQDPT}^{b}$ & & & \\
\hline $6-31 \mathrm{G}(\mathrm{d})$ & & & 3.66 \\
\hline
\end{tabular}

${ }^{a}$ present work

${ }^{b}$ Reference 66. 